Review - Part of the Special Issue: Alzheimer's Disease - Amyloid, Tau and Beyond

\title{
Perspective on future role of biological markers in clinical therapy trials of Alzheimer's disease: A long-range point of view beyond 2020
}

\author{
Harald Hampel $^{\text {a,* }}$, Simone Lista ${ }^{\text {b,* }}$, Stefan J. Teipel ${ }^{\text {c,d }}$, Francesco Garaci ${ }^{\text {e,f }}$, \\ Robert Nisticò $^{\text {g,h }}$, Kaj Blennow ${ }^{\mathrm{i}}$, Henrik Zetterberg ${ }^{\mathrm{i}, \mathrm{j}}$, Lars Bertram ${ }^{\mathrm{k}}$, Charles Duyckaerts ${ }^{1}$, \\ Hovagim Bakardjian $^{\mathrm{m}, \mathrm{n}}$, Alexander Drzezga ${ }^{\circ}$, Olivier Colliot ${ }^{\mathrm{p}, \mathrm{q}, \mathrm{r}, \mathrm{s}, \mathrm{t}}$, Stéphane Epelbaum $^{\mathrm{u}, \mathrm{v}}$, \\ Karl Broich $^{\mathrm{w}}$, Stéphane Lehéricy ${ }^{\mathrm{n}, \mathrm{p}, \mathrm{q}, \mathrm{r}, \mathrm{s}}$, Alexis Brice ${ }^{\mathrm{p}, \mathrm{q}, \mathrm{r}, \mathrm{s}, \mathrm{x}}$, Zaven S. Khachaturian ${ }^{\mathrm{y}}$, \\ Paul S. Aisen ${ }^{\mathrm{z}}$, Bruno Dubois ${ }^{\mathrm{u}, \mathrm{v}}$
}

${ }^{\text {a } U n i v e r s i t e ́ ~ P i e r r e ~ e t ~ M a r i e ~ C u r i e, ~ D e ́ p a r t e m e n t ~ d e ~ N e u r o l o g i e, ~ I n s t i t u t ~ d e ~ l a ~ M e ́ m o i r e ~ e t ~ d e ~ l a ~ M a l a d i e ~ d ' A l z h e i m e r(I M 2 A), ~ P a v i l l o n ~ F r a n c ̧ o i s ~ L h e r m i t t e, ~ H o ̂ p i t a l ~}$ de la Salpêtrière, Paris, France

${ }^{\mathrm{b}}$ Department of Psychiatry, Psychotherapy and Psychosomatics, Martin-Luther-University Halle-Wittenberg, Halle/Saale, Germany

${ }^{\mathrm{c}}$ Department of Psychosomatic Medicine, University of Rostock, Rostock, Germany

${ }^{\mathrm{d}}$ German Center for Neurodegenerative Diseases (DZNE) Rostock/Greifswald, Rostock, Germany

e Department of Diagnostic Imaging, Molecular Imaging, Interventional Radiology, and Radiotherapy, University of Rome "Tor Vergata", Rome, Italy

f IRCCS San Raffaele Pisana, Rome and San Raffaele Cassino, Cassino, Italy

${ }^{g}$ Department of Physiology and Pharmacology, University of Rome "La Sapienza", Rome, Italy

${ }^{\mathrm{h}}$ IRCSS Santa Lucia Foundation, Rome, Italy

institute of Neuroscience and Physiology, Department of Psychiatry and Neurochemistry, The Sahlgrenska Academy at the University of Gothenburg, Mölndal, Sweden

${ }^{\mathrm{j}}$ University College London Institute of Neurology, Queen Square, London, UK

${ }^{\mathrm{k}}$ Department of Vertebrate Genomics, Max Planck Institute for Molecular Genetics, Berlin, Germany

${ }^{1}$ Laboratoire de Neuropathologie Raymond-Escourolle, Groupe Hospitalier Pitié-Salpêtrière, AP-HP, Paris, France

${ }^{m}$ IM2A - Institute of Memory and Alzheimer's Disease, Paris, France

${ }^{\mathrm{n}}$ IHU-A-ICM - Paris Institute of Translational Neurosciences Pitié-Salpêtrière University Hospital, Paris, France

${ }^{\circ}$ Department of Nuclear Medicine, University Hospital of Cologne, Cologne, Germany

${ }^{\mathrm{P}}$ Université Pierre et Marie Curie-Paris 6, Centre de Recherche de l'Institut du Cerveau et de la Moelle Épinière, UMR-S975 Paris, France

${ }^{\mathrm{q}}$ Inserm, U975, Paris, France

${ }^{\mathrm{r}}$ CNRS, UMR 7225, Paris, France

${ }^{\mathrm{S}}$ ICM - Institut du Cerveau et de la Moelle Épinière, Paris, France

${ }^{\mathrm{t}}$ INRIA, Aramis Team, Centre de Recherche Paris-Rocquencourt, France

u'Institut de la Mémoire et de la Maladie d'Alzheimer (IM2A), Département de Neurologie, Hôpital de la Pitié Salpêtrière, Paris, France

${ }^{v}$ Université Pierre et Marie Curie, Paris, France

${ }^{\mathrm{w}}$ Federal Institute of Drugs and Medical Devices (BfArM), Bonn, Germany

${ }^{x}$ AP-HP, Hôpital de la Salpêtrière, Département de Génétique et Cytogénétique, Paris, France

' The Campaign to Prevent Alzheimer's Disease by 2020 (PAD2020), Potomac, MD, USA

${ }^{\mathrm{z}}$ Department of Neurosciences, University of California, San Diego, San Diego, CA, USA

\section{A R T I C L E I N F O}

\section{Article history:}

Received 3 October 2013

Accepted 13 November 2013

Available online 22 November 2013

\section{Keywords:}

Alzheimer's disease

CSF/blood biomarkers

Structural/functional MRI markers

PET imaging markers

Clinical trials

\begin{abstract}
A B S T R A C T
Recent advances in understanding the molecular mechanisms underlying various paths toward the pathogenesis of Alzheimer's disease (AD) has begun to provide new insight for interventions to modify disease progression. The evolving knowledge gained from multidisciplinary basic research has begun to identify new concepts for treatments and distinct classes of therapeutic targets; as well as putative disease-modifying compounds that are now being tested in clinical trials.

There is a mounting consensus that such disease modifying compounds and/or interventions are more likely to be effectively administered as early as possible in the cascade of pathogenic processes preceding and underlying the clinical expression of AD. The budding sentiment is that "treatments" need to be applied before various molecular mechanisms converge into an irreversible pathway leading to morphological, metabolic and functional alterations that characterize the pathophysiology of AD. In light
\end{abstract}

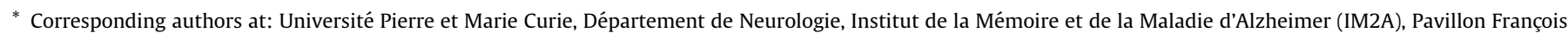
Lhermitte, Hôpital de la Salpêtrière, 47 Boulevard de l'Hôpital, 75013 Paris, France. Tel.: +33 0142162128 ; fax: +33 0981123444.

E-mail addresses: harald.hampel@psl.aphp.fr (H. Hampel), slista@libero.it (S. Lista).
} 
of this, biological indicators of pathophysiological mechanisms are desired to chart and detect AD throughout the asymptomatic early molecular stages into the prodromal and early dementia phase.

A major conceptual development in the clinical AD research field was the recent proposal of new diagnostic criteria, which specifically incorporate the use of biomarkers as defining criteria for preclinical stages of AD. This paradigm shift in AD definition, conceptualization, operationalization, detection and diagnosis represents novel fundamental opportunities for the modification of interventional trial designs.

This perspective summarizes not only present knowledge regarding biological markers but also unresolved questions on the status of surrogate indicators for detection of the disease in asymptomatic people and diagnosis of $\mathrm{AD}$.

(c) 2013 Elsevier Inc. All rights reserved.

\section{Contents}

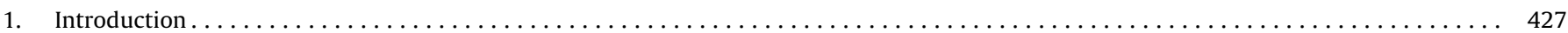

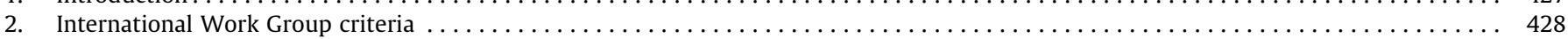

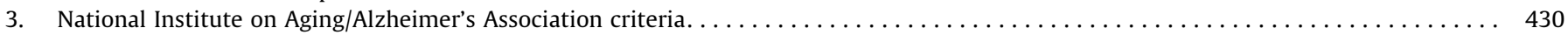

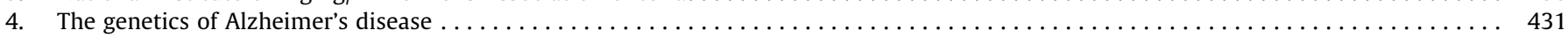

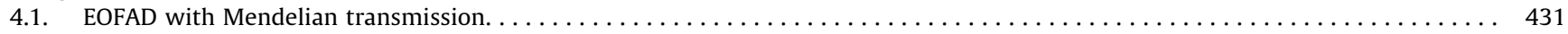

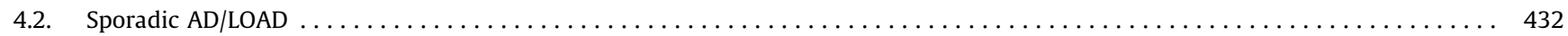

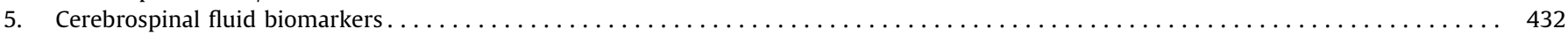

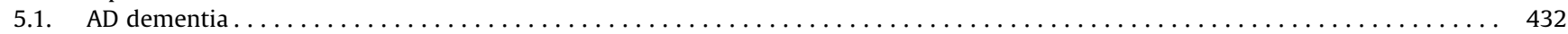

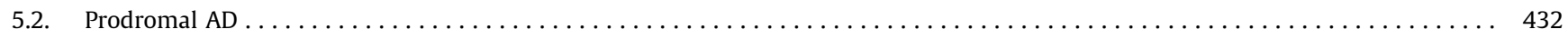

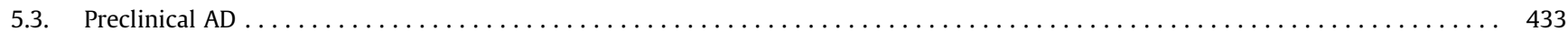

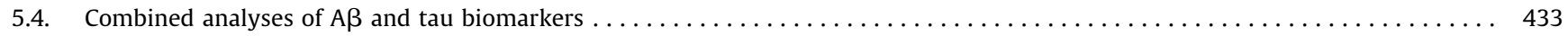

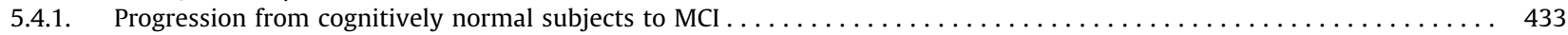

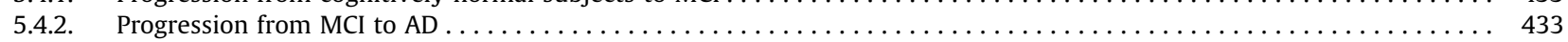

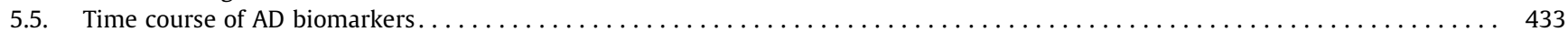

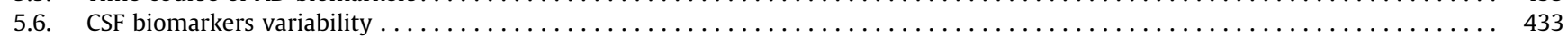

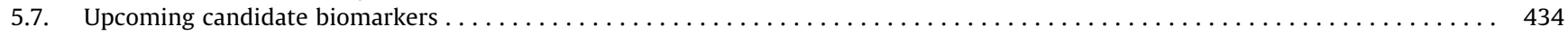

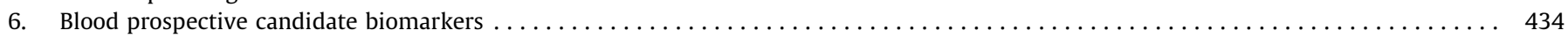

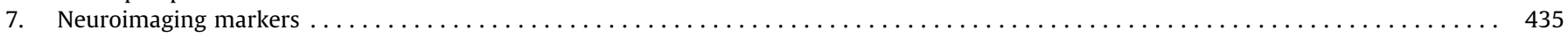

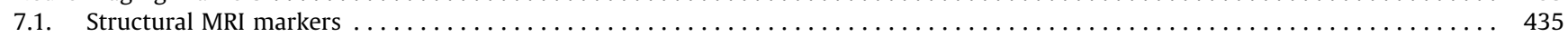

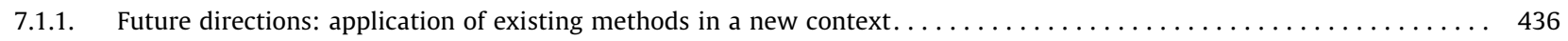

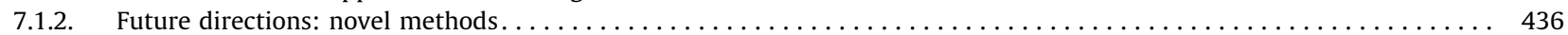

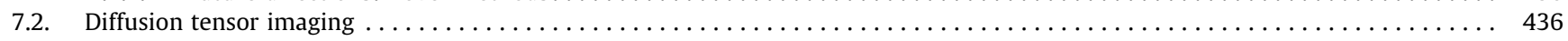

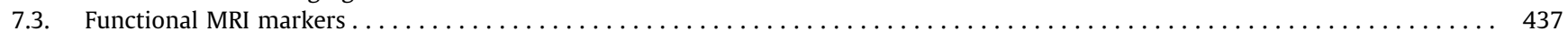

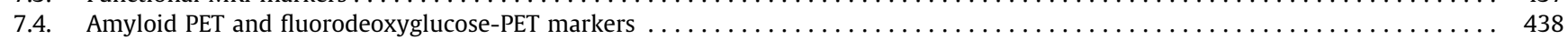

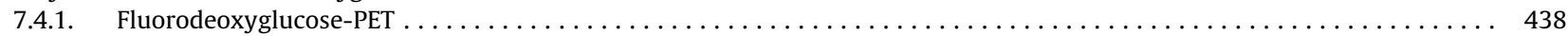

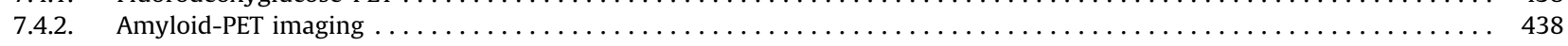

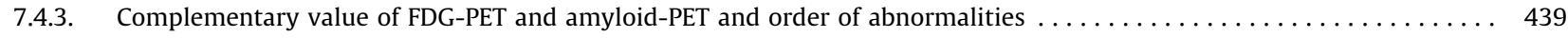

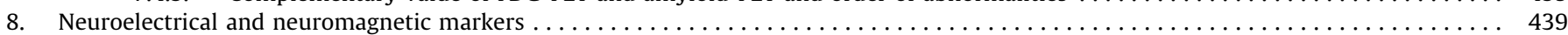

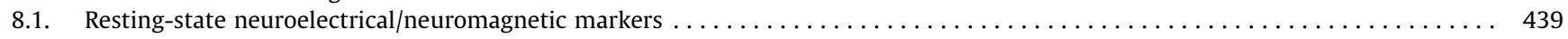

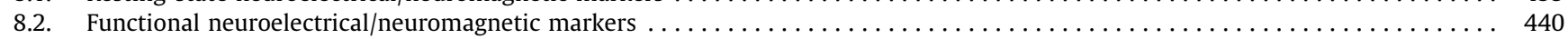

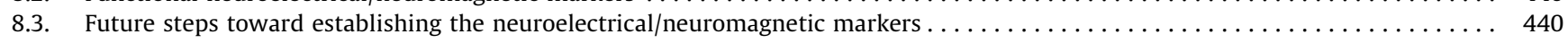

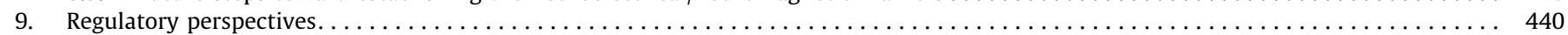

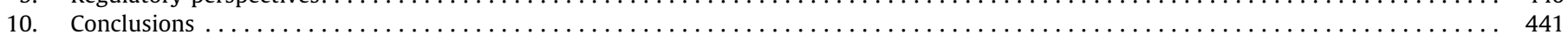

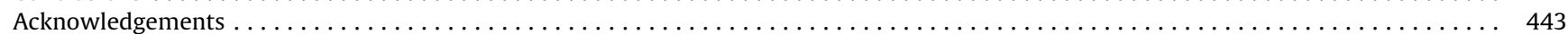

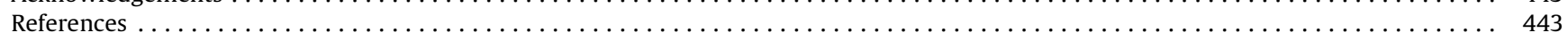

\section{Introduction}

Sporadic Alzheimer's disease (AD) is currently conceptualized as a multifactorial neurodegenerative disease transitioning later through a prodromal cognitive stage into a late-stage dementia syndrome. This initially clinically "silent" multi-dimensional disease cascade chronically, non-linear progressively unfolds through the emergence and probably at some point convergence of a yet not fully understood and characterized parallelized and/or interrelated array of molecular mechanisms and signaling pathways. For many decades, the definite diagnosis of AD has relied on the postmortem detection of senile plaques (SPs) and neurofibrillary tangles (NFTs). There, these historic hallmark neuropathological lesions have been extensively studied. Their molecular constituents have been isolated (intracellular aggregation of tau protein and extracellular accumulation of amyloid beta $(A \beta)$ peptide). The neuropathology is now better understood in terms of amyloid and tau pathology - as a consequence $A \beta$ and tau assays having secondarily been developed and validated during the last two decades to provide first "core feasible" cerebrospinal fluid (CSF) biomarkers. The stereotyped progression of tau [1] and $A \beta$ pathology [2] in the brain has been described and is the basis of the new National Institute on Aging and the Alzheimer's Association neuropathological criteria [3]. The amyloid cascade hypothesis, relying on the observation that all the mutations causing early-onset $\mathrm{AD}$ involve genes that alter $\mathrm{A} \beta$ production, has generated a theory emphasizing the central relevance of the amyloidogenic cascade and the $A \beta$ peptide. As a consequence, many treatment trials in $A D$ have been aimed at altering the abnormal production, accumulation and deposition $A \beta$. The optimism that reducing $A \beta$ accumulation and/or 
deposition would directly result into an improved clinical and functional patient status, however, has not yet been fulfilled. Recent evidence indicating that misfolding of $A \beta$ and tau could be transmitted to normal proteins of the host through brain injections of affected samples is hypothesis generating and opens new translational research perspectives. A mono-linear amyloid cascade perspective, would seem reductionistic, since it fails to recognize the role of the many conformations that the proteins may adopt, explaining the progression of the disease through the connections and the transmissibility of the pathology in some experimental conditions. Therefore, in the advent of the worldwide AD epidemic, critical reassessment of the evidence-based significance and limitation of prevailing as well as of emerging fundamental concepts of AD pathophysiology seems to be necessary to foster breakthrough advances to effectively detect, treat or even prevent AD [4].

The search for biomarkers of preclinical $\mathrm{AD}$ is becoming increasingly important because pathogenesis-targeted neuroprotective strategies are being developed for future use in "at risk" populations. Advances in new neuroimaging probes and technologies, identification of new biochemical markers of AD in plasma, blood and CSF, and breakthroughs in molecular genetics and basic neuroscience are gradually translating into better understanding of predisposing and preclinical factors that lead to progressive neurodegeneration and finally cognitive and behavioral symptoms and dementia.

At present, the combination and integration of multimodal imaging methods, neurochemical markers, and genetic strategies are still in their infancy. However, significant indications on the existing state of the biomedicine on candidate markers of $\mathrm{AD}$ resulting from multiple analytical platforms - encompassing (I) structural/functional/metabolic neuroimaging modalities, (II) neurochemistry methods based on CSF and blood (plasma/serum) examination, (III) neurogenetic analyses, and (IV) procedures for cognitive and functional assessment - have been supplied [5-13].

The next-generation of studies is required to use multicenter data sets that exploit the large variety of affected systems to appraise the stability of multimodal diagnostic algorithms in a multinational multicenter setting. A growing number of national and international platforms are following this central line of research, among them the US Alzheimer's Disease Neuroimaging Initiative (US-ADNI) [14] and the European ADNI (E-ADNI) [15] that, in conjunction with other parallel projects around the globe, are collectively known as Worldwide ADNI (WW-ADNI) [16]. The ADNI has been designed to validate neuroimaging, CSF, and bloodplasma biomarker candidates for $\mathrm{AD}$ treatment trials, and therefore aid and speed drug development [16]. As a result, the approach of combining different source markers might be of help in the identification of those subjects who will develop AD and who are consequently potential targets for prevention as well as symptomatic pharmacological interventions.

When employed in $\mathrm{AD}$ clinical trials, biomarkers can be utilized: (I) to improve the diagnostic accuracy in trial participants, enabling patient cohorts to be enriched with characteristic molecular mechanisms of AD; (II) for stratification of AD patients; (III) for safety monitoring, i.e. to assess and predict tolerability and adverse side effects; (IV) as theragnostic markers, i.e. to identify and monitor the biochemical effects of drugs [5,6,17]. Notably, biomarkers provide the potential for characterization and validation of drug mechanisms of action, monitoring AD course and progression, and evaluating therapeutic response/outcome [18]. Furthermore, since biomarker profiles reflect different stages of the pathogenic process, they can be utilized to recruit optimal individuals for trials of different drugs and different clinical phenotypes at different stages of AD pathophysiology [19].

By using multimodal strategies, $\mathrm{AD}$ has been categorized into different stages according to the presence of biomarkers and the patterns of cognitive impairment. Following a pre-pathology stage characterized by normal biomarkers and absence of cognitive impairment, AD dimensionally (not categorically) emerges exhibiting through an asymptomatic stage (biomarkers abnormal, no cognitive impairment) subsequently to a symptomatic stage (biomarkers abnormal, cognitive impairment) that can be further differentiated into a subjective cognitive impairment $(\mathrm{SCI})$ stage (AD-SCI), a prodromal, often categorized as a "mild cognitive impairment" (MCI) stage (AD-MCI), and finally a syndromal dementia stage (AD-dementia) [20]. Notably, these categories are mere restrictive research or practical clinical constructs and should not mask the true continuous dimensional character of AD.

The present review will summarize the current knowledge on the employment of biological markers in $\mathrm{AD}$ and provide perspectives as well as future directives on major areas of $A D$ biomarker discovery and development emphasizing the role of such markers for use in clinical trials. Notably, since this manuscript is intended to raise evolving debate on the effective discovery, development, validation, and qualification process of biological markers resulting from all available technical modalities, it represents a major complement and extension to the antecedent perspective by Hampel et al. [7]. Current knowledge and perspectives/future directives on the employment of biological markers in AD are summarized in Tables 1 and 2, respectively.

\section{International Work Group criteria}

In 2007, an International Work Group (IWG) led by Dubois and colleagues has provided a novel description of $\mathrm{AD}$ as a clinicobiological syndrome that can be documented in vivo, prior to the onset of dementia, by a "core" clinical phenotype that includes an amnestic syndrome of the hippocampal type and indication from biomarkers reflecting the existence of Alzheimer-type pathology [21]. Such criteria may be used throughout any phase of the AD spectrum after the beginning of clinical signs [22]. Moreover, a specific terminology has been developed to resolve issues related to $\mathrm{AD}$ reconceptualization [23].

The IWG proposed two new sets of diagnostic criteria requiring the assessment of AD biomarkers. The first, covering asymptomatic AD individuals, is defined "preclinical AD". Preclinical AD has been then partitioned into the "asymptomatic at risk for $A D$ " and the "presymptomatic AD" categories [23], the latter applying to asymptomatic individuals who carry familial autosomal dominant $\mathrm{AD}$ mutations. The second group, applying to symptomatic AD individuals, is designated as "AD". Individuals reflecting these criteria can be, in turn, categorized into "prodromal AD" (or "predementia AD") and "AD dementia" [23].

The most important progress inherent in the IWG criteria is the integration of biomarkers into a diagnostic scheme that allows a biology aided assessment of AD which is integrated with the clinical signs and symptoms, independent of disease severity. The use of biomarkers is integral to the diagnosis of AD in the IWG criteria; consequently, the presence of pathophysiologic or topographic aberrations representative of $\mathrm{AD}$ is strictly required. The pathophysiologic markers encompass the molecular signatures of $A D$ in the CSF (low levels of the 42 amino acid-long form of the $A \beta$ peptide $\left(A \beta_{1-42}\right)$ plus increased concentrations of total-tau (t-tau) and/or hyperphosphorylated tau (phospho tau, p-tau) proteins) or significant binding of amyloid ligands using positron emission tomography (PET). The topographic markers consist of medial temporal/ hippocampal atrophy on magnetic resonance imaging (MRI) or bilateral parieto-temporal hypometabolism on PET [22].

Importantly, the IWG criteria have abandoned the categorical concept of "MCl", which is heterogeneous in terms of AD progression and has many different underlying causes, in favor 
Table 1

Current knowledge on the employment of biological markers in AD.

\begin{tabular}{|c|c|}
\hline Area of markers & Key points \\
\hline \multicolumn{2}{|l|}{ Genetics } \\
\hline Familial AD & $\begin{array}{l}\text { - "Featured genes" (causal genes): APP, PSEN1, PSEN2 } \\
\text { - Currently known mutations in APP, PSEN1, PSEN2 genes do not account for all Mendelian AD cases, suggesting the existence of AD- } \\
\text { causing mutations in other genes }\end{array}$ \\
\hline Sporadic AD & $\begin{array}{l}\text { - "Featured genes" (proposed susceptibility genes): APOE, BIN1, CLU, ABCA7, CR1, PICALM, MS4A6A, MS4A4E, CD33, CD2AP, EPHA1, TREM2 } \\
\text { and counting } \\
\text { - The advent of GWAS have led to the identification of novel loci linked to mostly LOAD risk } \\
\text { - These genes appear to be mostly linked with three molecular pathways: (I) the amyloidogenic cascade, (II) cholesterol-lipid } \\
\text { metabolism, and (III) immune-inflammatory mechanisms }\end{array}$ \\
\hline
\end{tabular}

Cerebrospinal fluid

- CSF biomarkers $A \beta_{1-42}$, t-tau, p-tau ${ }_{181}$, and p-tau ${ }_{231}$ have a high diagnostic accuracy for $A D$, and for prodromal $A D$ in patients with $\mathrm{MCI}$ - CSF levels of $A \beta_{1-42}$ start declining in the preclinical phase of sporadic $A D$, prior to any evident increase in t-tau or p-tau

- CSF biomarkers, especially $A \beta_{1-42}$, convert to pathologic values several years before the first appearance of clinical signs, also in the familial form of AD

- The diagnostic accuracy for the combination of CSF $A \beta_{1-42}$, t-tau, and p-tau has been reported to be higher than for any biomarker alone

- CSF biomarkers are increasingly used in clinical trials, both for enrichment of patient populations with pure AD cases at the inclusion and to evaluate the biochemical effects of treatment (theragnostic markers)

- CSF biomarker $A \beta_{1-42}$ is the central CSF biomarker for $A \beta$ metabolism and deposition in clinical treatment trials.

- CSF biomarkers t-tau and p-tau are the central CSF biomarkers to monitor the intensity of cortical axonal degeneration and tau phosphorylation state, respectively, in clinical treatment trials

Blood - Definite data regarding the association of plasma $A \beta_{1-40}$ and $A \beta_{1-42}$ concentrations with incipient $A D$ are presently lacking

- The development of mass spectrometry-based technologies has elected proteomics as the chief platform to inspect the plasma/serum proteome for the discovery of next-generation biomarkers showing diagnostic, prognostic, or therapeutic efficacy

- Blood-based profiles/signatures including panels of molecules related to immune regulation and inflammatory pathways have been discovered

- Issues in plasma/serum proteomics, including pre-analytical variables, requiring standardization for specimen collection/processing, quantitation, and setting strategies for managing biomarkers after their detection, currently exist. These markers do not seem to be ready for clinical applications

Structural neuroimaging

- Reduction in hippocampus volumetry, derived from structural MRI, has been consistently found in AD and MCI across a wide range of mono- and multicenter studies

- Hippocampus volumetry has also been used as a secondary endpoint in clinical trials on potential disease modifiers in AD or MCI

- The EMA regulatory authorities have endorsed a qualification process for the use of low hippocampus volume for enrichment of study samples

- Few automated protocols have already been cleared for marketing as a medical device by the FDA

- The attractiveness of MRI as endpoint in clinical trials is related to the assumption that regional brain volume can serve as in vivo surrogate of neuronal density

- Neuropathological evidence suggests a selective involvement of specific subcortical areas, most notably the cholinergic nuclei of the basal forebrain and noradrenergic nuclei in the locus coeruleus in $\mathrm{AD}$

- Diffusion Tensor Imaging has become a leading method in investigating white matter microarchitecture and integrity and has been widely employed in $\mathrm{AD}$ and $\mathrm{MCI}$

Functional neuroimaging

- Functional MRI, studying the neuronal activity through non-invasive means during specific cognitive states, has been able to detect functional alterations prior to onset of cognitive impairment or AD-related structural neurodegeneration

- Functional MRI studies are focused on the "default mode network", i.e. the interplay between a set cortical areas and the hippocampal memory system

In vivo molecular neuroimaging - FDG-PET has demonstrated to be of great value because it allows the detection of different patterns of neurodegeneration; it is also highly useful in differentiating within amyloid-positive subtypes of disease which cannot be distinguished on the basis of their amyloid PET-scan

- $\mathrm{PiB}$ is the current gold-standard tracer for PET amyloid-imaging. Recently, ${ }^{18} \mathrm{~F}$-labeled compounds have been evaluated to enable allow a more widespread application of this method. $\left[{ }^{18} \mathrm{~F}\right]$ Florbetapir/Amyvid has already been approved by the FDA and the EMA - Concerning early diagnosis, several studies demonstrated a high predictive value of a positive amyloid-scan in the stage of MCI with regard to conversion to $\mathrm{AD}$

Neurodynamics

Resting state

- Time-resolved EEG and MEG measures have been increasingly explored to identify predementia $\mathrm{AD}(\mathrm{MCI})$

- Brainwave band power estimates in the delta, theta, alpha, beta and gamma frequency bands, as well as their ratios, have been used as a major tool to demonstrate RSN changes in AD and predementia AD patients as compared to healthy controls

- Studied have shown a connection between clinical (MMSE) measures and frequency band power (alpha)

Functional

- ERP/ERF markers (peak latency, amplitude, brain sources) measure task-related functional changes which are not available in resting state. Deterioration of cognitive/episodic memory measures ( $\mathrm{P} 300, \mathrm{P} 600$, etc.) has been demonstrated in $\mathrm{AD}$ and predementia $\mathrm{AD}$ subjects by multiple studies

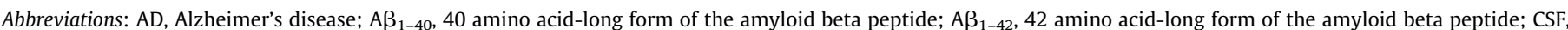

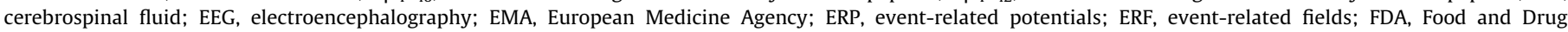

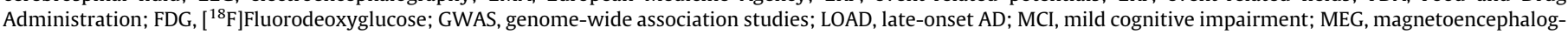

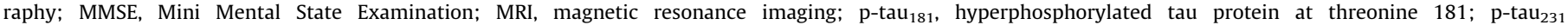

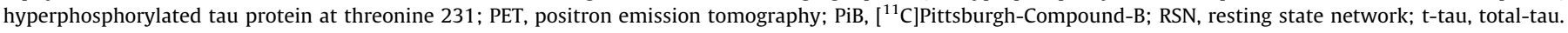

of prodromal AD in subjects showing symptomatic predementia AD. Therefore, these criteria rely on the implementation of biomarkers to detect a specific subset of $\mathrm{MCI}$ individuals who are in the predementia phase $[22,24]$.
From a conceptual perspective, the IWG criteria foster the perception of $\mathrm{AD}$ as a dimensional clinico-biological entity and have been positively applied in clinical therapy trials approved by the US Food and Drug Administration (FDA) [22]. They have been 
Table 2

Short- to mid-term perspectives and future directives of biological markers in AD.

\begin{tabular}{|c|c|}
\hline Area of markers & Key points \\
\hline Genetics & $\begin{array}{l}\text { - Additional AD susceptibility variants are expected to be identified in upcoming GWAS based on larger sample sizes and/or higher } \\
\text { resolution genetic maps } \\
\text { - Resequencing part (e.g. by targeting specific functional regions such as the exome) or all of the human genome using "next-generation } \\
\text { sequencing" technologies } \\
\text { - "Next- and third generation sequencing" technologies will allow efficiently extending the knowledge of AD genetics to the lower allele } \\
\text { frequency spectrum, down to low-frequency variants }\end{array}$ \\
\hline
\end{tabular}

Cerebrospinal fluid • The use of multiple longitudinal CSF specimens is necessary to detect the time point at which CSF biomarkers convert from physiologic to pathologic values

- Substantial progresses in the exploratory "omics" disciplines, especially proteomics/metabolomics, will enhance the detection of novel candidate CSF biomarkers

- Many candidate biomarkers have the potential to increase the diagnostic accuracy of the "core" biomarkers $A \beta_{1-42}, t-t a u$, and p-tau (e.g. BACE1)

Blood • Progress in blood-based biomarker discovery relies on the establishment of Standard Operating Procedures (SOPs) for the appropriate selection of patients and specimens

- The Human Plasma Proteome Project (HPPP) is an initiative launched that will face matters related to pre-analytical variability and to make attempts to establish SOPs

- The Blood-Based Biomarker Interest Group (BBBIG), an international working group of leading AD scientists from academia and industry, will inspect the present scenario of biomarker discovery in blood in order to identify current needs that will enable the field to progress

- It seems to be doubtful that a blood marker alone will be in itself adequate for the diagnosis of $\mathrm{AD}$

- In contrast, it seems most likely to have combinations of markers: several proteins coupled with other blood-based or non-bloodbased markers, such as imaging

Structural neuroimaging • The EADC-ADNI hippocampal harmonization project is providing an internationally consented protocol for manual hippocampus segmentation that will serve to validate automated hippocampus volumetry methods

- Future studies are needed to address more specifically associations between regional brain atrophy pattern and regional markers of neuronal degeneration

- The next years will see increasing use of automated volumetry of hippocampus or regional brain atrophy pattern as secondary endpoints in clinical trials in prodromal $\mathrm{AD}$ and $\mathrm{AD}$ dementia stages

- Structural imaging markers are being used to enrich the risk for AD in clinical samples of MCI subjects for clinical trials. In addition, structural MRI will help to enrich study samples of asymptomatic subjects with positive molecular biomarkers of AD

- The presence of hippocampus atrophy together with amyloid positivity will help to select subjects with a high risk of conversion to AD or MCI within a timeframe that is relevant for a clinical trial

- The effect of a novel structural imaging marker of predementia AD on care systems worldwide, that have difficulties to provide adequate care even to patients in clinically manifest stages of disease, needs to be assessed in future studies

- Novel methods, including high-field MRI at 3 Tesla and ultra-high field MRI at 7 Tesla, will gain increasing importance to understand the morphological/neuroanatomical basis of cognitive decline in $\mathrm{AD}$

- Based on mappings of subcortical nuclei from postmortem analyses, MRI scans in cranio will help to identify early changes in cholinergic and noradrenergic projecting nuclei in predementia and dementia stages of $\mathrm{AD}$

- MRI-based detection of amyloid plaques in humans will become a major topic of research in coming years. The use of 7 Tesla MRI in human studies may allow in vivo detection of cortical amyloid deposition in the future

Functional neuroimaging

- Functional MRI will be increasingly applied in the area of novel pharmaceutical strategies, in AD and MCI. Although drug-induced modulation of memory-related networks have been detected by functional MRI, few studies have demonstrated abnormal activation following pharmacological treatment in $\mathrm{MCI}$ and $\mathrm{AD}$

In vivo molecular neuroimaging • Novel imaging instrumentation such as hybrid PET/MRI scanners may offer the opportunity to merge the complementary information from different imaging modalities into new integrated in vivo biomarkers of neurodegeneration

Neurodynamics

- Design of enhanced EEG/MEG-based AD biomarkers:

- Neurodynamic measures (such as brain connectivity, global synchronization, synchronization likelihood, detrended fluctuation analysis, approximate entropy, mutual information, source localization, and other non-linear signal features) will be used within the framework of both the resting-state and functional biomarker paradigms to adapt better to the complex characteristics and dynamics of progressive neurodegeneration and aging

- Future functional EEG/MEG biomarkers will rely on multidimensional (spatio-spectro-temporal characteristics) in order to handle efficiently single-trial EEG/MEG data and increase sensitivity/specificity

- Efficient biomarker selection with the final goal to evaluate the current state of AD-related functional brain networks for each individual subject

- Standardization and validation of selected EEG/MEG-based AD biomarkers:

- A selected battery promising neurodynamic biomarkers will pass through a rigorous multi-step and multi-center standardization/ validation process before they can be used as diagnostic aids

- Modular approach will be required for new biomarker standards. A robust review procedure will be put in place to facilitate fast and efficient biomarker upgrades

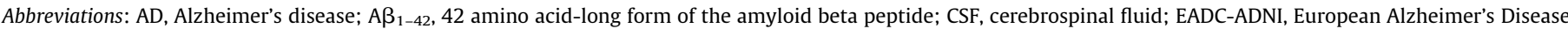

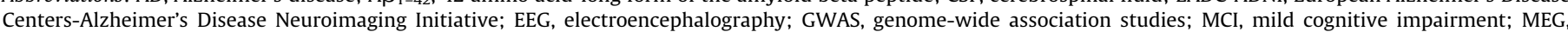
magnetoencephalography; MRI, magnetic resonance imaging; p-tau, hyperphosphorylated tau protein; PET, positron emission tomography; t-tau, total-tau.

recognized by the European Medicine Agency (EMA) [25] for the employment in clinical drug trials as well.

\section{National Institute on Aging/Alzheimer's Association criteria}

Following the emerging development of the IWG/Dubois criteria, the National Institute on Aging (NIA) and the Alzheimer's
Association (AA) summoned three working parties aimed at establishing criteria for the staging of AD [26-28]. Differently from the IWG that use an integrated clinico-biological approach covering all of the AD symptomatic phases, the NIA-AA employs three different categories of criteria for cases in which biomarkers have been measured: one for the asymptomatic phase ("preclinical $\mathrm{AD}$ "), one for the AD-MCI phase ("MCI due to AD"), and one for the 
AD-dementia phase ("dementia due to AD") [20]. Notably, the NIAAA criteria distinguish between amyloid and neuronal injury markers. This distinction is based on the hypothesis that $A \beta$ generation drives other pathophysiological changes, an idea strongly supported by genetic evidence from familial autosomal dominant $\mathrm{AD}$, Down's syndrome, and the recent demonstration of a protective mutation in the amyloid precursor protein $(A P P)$ gene [29]. The biomarkers of $A \beta$ accumulation are represented by significant amyloid tracer retention using PET imaging and/or low CSF concentrations of $A \beta_{1-42}$. The biomarkers of neuronal degeneration or injury consist of increased levels of CSF tau ( $t$ tau or p-tau), reduced fluorodeoxyglucose uptake on PET in specific areas encompassing temporoparietal cortex, and atrophy on structural MRI primarily including medial temporal lobes and parietal cortices [30,31].

Subjects with preclinical AD can be categorized into three stages using cognitive markers and biomarkers. In particular, individuals showing only anomalous amyloid markers are classified in stage 1; those with both atypical amyloid and injury markers are considered in stage 2 ; those showing both unusual amyloid and injury markers accompanied by minimal cognitive impairments, such as $\mathrm{SCI}$, are classified in stage 3. Individuals with $\mathrm{MCI}$ due to $\mathrm{AD}$ or dementia due to $\mathrm{AD}$ are categorized in a risk staging model according to amyloid and neuronal injury markers, as follows: (I) high likelihood for $\mathrm{AD}$ if both amyloid and neuronal injury markers are aberrant, (II) intermediate likelihood for AD if only one of the two markers has been assessed and is anomalous, (III) uninformative if one marker is atypical and the other normal, or vice versa [20].

The IWG group considers the presence of brain amyloid accumulation in the absence of clinical features in the sporadic population to be indicative of an "at risk" group. In contrast, the NIA-AA group considers such individuals to indeed already have preclinical $A D$, suggesting that in time they would develop cognitive decline and the clinical dementia syndrome. This presents a fundamental hypothetical and conceptual difference of the two approaches with practical consequences for trials which needs to be further elucidated.

\section{The genetics of Alzheimer's disease}

$\mathrm{AD}$ has been designated as a multifaceted pathology characterized by a high-degree of genetic heterogeneity. This implies both that the same phenotype can be generated or modified by a number of different genetic loci and alleles, and that mutations or polymorphisms at different positions in the same gene lead to the same clinical syndrome [32]. This situation is aggravated by the fact that, in some instances, different mutations in the same gene can lead to clinically distinct syndromes. Hence, AD is considered to belong to the growing fraction of "genetically complex" diseases.

A peculiar feature observed in AD is the dichotomy of (I) familial versus (II) "apparently" non-familial forms of disease. The former, referred to as familial $A D$, accounts for less than $5 \%$ of all $A D$ cases and is often conferred by individual disease-causing mutations transmitted in classic Mendelian fashion, mostly typically by autosomal dominant transmission. Since age of onset in these forms of $\mathrm{AD}$ is usually early ( $<65$ years) or very early ( $\leq 50$ years), it is often also called early-onset familial AD (EOFAD). The latter, commonly defined as non-Mendelian, "polygenic", or "sporadic" $A D$, accounts for about $95 \%$ of all $A D$ cases. It is typically characterized by an onset age well beyond 65 years of age, and it is also designated as late-onset AD (LOAD) [32,33]. However, it should be highlighted that this dichotomization scheme is over simplistic, as there are cases of EOAD without evidence for familial clustering or Mendelian transmission while, on the other hand, these clustering and transmission patterns are frequently observed in LOAD [33]. In addition to these genetic causes, non-genetic (e.g. environmental or epigenetic) factors are likely significantly affecting an individual's risk to develop AD. However, the exact mechanisms underlying the possible pathogenic effects of these non-genetic factors are still mostly elusive which is, at least in part, owing to the fact that it is still relatively difficult to detect and evaluate them experimentally [34].

The introduction of high-throughput DNA genotyping and sequencing technologies, allowing to systematically screen the genomes of a large number of individuals simultaneously, has led to the completion of a high number of genome-wide association studies (GWAS) in AD. These studies allow simultaneously investigating literally millions of genetic markers (mostly socalled single-nucleotide polymorphisms, SNPs) in one experiment to assess their effect on disease risk, or quantitative phenotypes. Not unexpectedly, these GWAS have led to more reproducible and more consistent findings than three decades of candidate-genedriven research before [35].

\subsection{EOFAD with Mendelian transmission}

EOFAD is caused by rare and highly penetrant mutations in three genes, namely: amyloid precursor protein (APP, located at chromosome region 21q21.2), presenilin 1 (PSEN1, located at 14q24.3), and presenilin 2 (PSEN2, located at 1q42.13) [33]. Presently, more than 220 distinct disease-causing mutations have been discovered across these genes (for an up-to-date summary, see the Alzheimer Disease \& Frontotemporal Dementia Mutation Database (AD\&FTDMDB) at http://www.molgen.vib-ua.be/ADMutations/ [36]). Currently, over $30 \mathrm{AD}$-causing mutations have been reported in $A P P$, encoding for the precursor protein for $A \beta$. Interestingly, most of the APP mutations occur near the putative $\gamma$ secretase site between amino acidic residues 714 and 717 , suggesting that the $\gamma$-cleavage event of APP or its (dys)regulation are crucial for the development of AD [32]. The vast majority of EOFAD mutations are observed in PSEN1 located on chromosome 14. PSEN1 encodes for a highly conserved polytopic membrane protein, presenilin 1 , which is involved in mediating intramembranous, $\gamma$-secretase processing of APP to generate A $\beta$ peptides [37]. At present, the overall number of known AD-causing mutations in PSEN1 exceeds 180. Lastly, EOFAD is rarely caused by mutations in PSEN2 which encodes for presenilin 2, which represents another member of the presenilin family of proteins, displaying substantial homology to presenilin 1 , both at the genomic and protein level $[38,39]$. In summary, the currently known AD-causing mutations occur in three different genes located on three different chromosomes. Functionally, the proteins encoded by all three genes share a common biochemical pathway, i.e. the altered production of the $A \beta$ peptide. Together, these findings provide strong support for the "amyloid hypothesis" indicating that an abnormal production and/or regulation of $A \beta$ is one of the main factors underlying AD pathogenesis [40]. While the currently known mutations in these three EOFAD genes account for a large fraction of Mendelian $\mathrm{AD}$, they do not account for all cases, suggesting that $\mathrm{AD}$-causing mutations in other genes exist. The successful identification of these hitherto unknown Mendelian AD genes could, thus, provide entirely new insights into $A D$ pathogenesis [33].

Recently, a study has detected mutations in the SORL1 gene in EOFAD patients [41]. SORL1 encodes for the protein SorLA that belongs to a set of protein-trafficking molecules in the endocytic and retromer pathways and is implicated in modulating the production of $A \beta$ peptide [41]. These findings suggest that SORL1 may represent a genetic risk factor for $A D$, although these data need independent replication. 


\subsection{Sporadic $A D / L O A D$}

In contrast to EOFAD, LOAD exhibits a significantly more complex and intricate pattern of interplay between genetic and non-genetic factors. This situation, combined with the fact that each factor only exhibits exceedingly small effect sizes, has been proven to make the identification of these factors a complicated issue.

The earliest and by far best established genetic risk factor for LOAD is the presence of one or two copies of the $\varepsilon 4$ allele in the apolipoprotein E gene (APOE), located on chromosome 19q13.2 [42]. The risk effect of $A P O E \& 4$ has been replicated in many studies across various ethnic groups. Besides the increase in $A D$ risk conferred by the $\varepsilon 4$ allele, a less pronounced protective effect has been reported, albeit somewhat less consistently, for the least common $\varepsilon 2$ allele [43]. Despite its comparatively large effect size, it is important to note that the presence of the APOE $\& 4$ allele is neither necessary nor sufficient to actually cause AD. Instead, it works as a bona fide genetic risk modifier, likely by diminishing the age of onset in a dose-dependent manner. In spite of the accomplishments of over two dozen published GWAS in AD, APOE $\varepsilon 4$ remains to be the single most important genetic risk factor for AD, both in terms of effect size and statistical significance [32].

Despite its well-known genetic association, the biochemical aspects of $A P O E \& 4$ in $A D$ pathogenesis are still only incompletely understood. The encoded protein, apolipoprotein $E$ (apoE), is synthesized in a large number of tissues, primarily in the liver. Hepatic apoE accounts for roughly three-quarters of circulating plasma levels of the protein $[44,45]$. The human brain is the second most prominent site of synthesis, chiefly occurring in the astrocytes [46] and microglia [47]. There is experimental evidence from transgenic mice that the expression of the human $\varepsilon 4$ allele and mutant APP promotes $A \beta$ accumulation during the course of the disease, suggesting that amyloid may accumulate progressively with time [48]. Moreover, apoE participates in cholesterol transport and lipid metabolism and, in addition to $\mathrm{AD}$, the $\varepsilon 4$ allele also represents a confirmed risk factor in vascular disease, likely owing to its link to augmented plasma cholesterol levels [49]. Amyloid angiopathy involving capillaries is much more prevalent in $A P O E$ \&4 carriers [50].

After the original report suggesting $A P O E \varepsilon 4$ to be a genetic risk factor in $\mathrm{AD}$, literally hundreds of genes have been investigated for evidence of genetic association and disease risk, mostly to no avail (for an up-to-date overview of the accumulated evidence, see the AlzGene database at http://www.alzgene.org/ [51]). As outlined above, this situation changed substantially with the advent of GWAS which have led to the identification of at least ten novel loci linked to mostly LOAD risk: BIN1, CLU, ABCA7, CR1, PICALM, MS4A6A, MS4A4E, CD33, CD2AP, and EPHA1 [52-56]. Functionally, these genes appear to be mostly linked with three (interdependent) molecular pathways: (I) the amyloidogenic cascade, (II) cholesterol-lipid metabolism, and (III) immune-inflammatory mechanisms [57]. Extending these leads, Jones et al. (2010) have assessed the functional role of SNPs not quite reaching genomewide significance in $\mathrm{AD}$ and arrived at a very similar conclusion, i.e. that especially pathways related to immune system response and lipid metabolism appear to be particularly overrepresented [58]. More recently, rare amino-acid changing variants in TREM2 (encoding for the triggering receptor located on myeloid cells 2) have been implicated as additional risk factors for $\operatorname{LOAD}[59,60]$. Intriguingly, the protein encoded by TREM2 is an immune receptor participating in the clearance of neural debris from the central nervous system (CNS), via processes including phagocytosis and reactive oxygen species production [61]. In all likelihood, additional AD susceptibility variants will be identified in upcoming GWAS based on larger sample sizes and/or higher resolution genetic maps. Equally, efforts are already under way to resequence part (e.g. by targeting specific functional regions such as the exome) or all of the human genome using "next-generation sequencing". Other than GWAS - which are based on microarray technology primarily targeting common genetic variations - these methods will allow efficiently extending our knowledge of $\mathrm{AD}$ genetics to the lower allele frequency spectrum, down to lowfrequency variants such as the ones already observed in TREM2. However, even the increasingly widespread application of these powerful new technologies will not abolish the need for extensive subsequent functional genetic experiments to elucidate the pathogenic mechanisms underlying the observed genetic effects [32].

\section{Cerebrospinal fluid biomarkers}

Owing to its contiguity to the brain parenchyma and the free exchange with the brain extracellular space, the biochemical composition of CSF is able to provide information on the brain chemistry. The distinctive features of CSF, together with the low incidence of complications after lumbar puncture [62] have supported the introduction of lumbar puncture and analyses of CSF biomarkers into routine clinical practice in some centers $[63,64]$. CSF biomarkers are also increasingly used in clinical drug trials, both for enrichment of the target population at inclusion and to evaluate the biochemical effects of treatment [65-67].

\subsection{AD dementia}

In the early' 90s, a first publication has documented elevated CSF amounts of t-tau in patients with AD dementia [68]. After that, augmented CSF concentrations of p-tau [69] and reduced levels of $A \beta_{1-42}$ [70] have been described. A large number of studies have replicated these findings. A decrease in CSF $A \beta_{1-42}$ to about $50 \%$ of the level in cognitively normal elderly subjects has been regularly reported, whereas an increase in CSF t-tau to approximately 300\% of the level in cognitively normal elderly subjects and a less evident growth in CSF p-tau to about 200\% have been repeatedly detected [71]. Such biomarkers show $80-95 \%$ of sensitivity and specificity in the dementia phase of the pathology $[71,72]$.

The CSF concentration of these markers is within the normal range in several differential diagnoses, including depression and Parkinson's disease $[5,69,72]$. Additionally, measurement of p-tau in CSF is of help to distinguish AD from other dementing pathologies, such as frontotemporal dementia and Lewy-body dementia. Only minimal differences among immunoassays specific for various epitopes of p-tau, including p-tau ${ }_{181}, \mathrm{p}-\mathrm{tau}_{231}$, and $\mathrm{p}$ $\mathrm{tau}_{199}$, have been found [73]. The diagnostic accuracy of these CSF biomarkers has also been substantiated in analyses in which the diagnosis was then proven by autopsy $[74,75]$ with comparable or superior discriminatory power than in studies utilizing patients with clinical diagnoses only.

\subsection{Prodromal $A D$}

CSF biomarkers exhibit a high predictive value in detecting prodromal $\mathrm{AD}$ in $\mathrm{MCI}$ subjects [72]. A study with a protracted clinical follow-up period has revealed that the combination of all three core CSF biomarkers shows a sensitivity of $95 \%$ to recognize prodromal AD in MCI [76]. Moreover, these markers are able to predict the rate of cognitive decline in patients with $\mathrm{MCI} /$ very mild AD dementia [77].

A high diagnostic accuracy of CSF biomarkers for prodromal AD has also been corroborated in large multicenter studies, such as the US-ADNI [75], the European Development of Screening guidelines and Criteria for Predementia Alzheimer's disease (DESCRIPA) study 
[78], and the Swedish Brain Power (SBP) project [79]. These findings emphasize the role of CSF biomarkers as clinical diagnostic tools to detect enhanced risk in $\mathrm{MCI}$ subjects to have prodromal AD.

\subsection{Preclinical $A D$}

The notion of preclinical AD designates cognitively normal subjects harboring early AD pathology, not severe enough to induce cognitive signs. The efficacy of CSF biomarkers in the preclinical stage to recognize patients who will progress to AD dementia has been assessed. Skoog et al. (2003) have found a reduction in CSF $A \beta_{1-42}$, but normal t-tau and p-tau levels in cognitively normal 85-year-olds who later developed dementia [80]. These results are corroborated in a population-based cohort of healthy elderly subjects aged 70-78 years with 8 years followup [81] and in a clinical study on asymptomatic elderly subjects aged 60-94 years [82]. According to these data, CSF levels of $A \beta_{1-42}$ start declining in the preclinical phase of sporadic $A D$, prior to any manifest increase in t-tau or p-tau.

With reference to familial AD, Moonis et al. (2005) have uncovered that asymptomatic subjects carrying familial $A D$ mutations exhibit both low CSF $A \beta_{1-42}$ and high t-tau concentrations [83]. This finding is confirmed in an analysis by Ringman et al. (2008) showing that mutation carriers have the full AD pattern of CSF biomarker changes long before symptom onset [84]. Bateman et al. (2012) have also suggested that CSF $A \beta_{1-42}$ may start to decrease already 25 years before the estimated clinical onset in familial AD mutation carriers, whereas increased CSF tau may be observed 15 years before predicted symptom onset [85]. Altogether, these results suggest that CSF biomarkers, especially $A \beta_{1-42}$, convert to positive several years before the first appearance of clinical signs, also in the familial form of the disease. Notably, familial AD mutation carriers - in their early 20s - may commence at higher $C S F A \beta_{1-42}$ concentrations than non-mutation carriers $[84,86]$. It should be noted that most of the studies published to date are "pseudo-longitudinal" in their design; they relate crosssectional biomarker data to longitudinal clinical or neuroimaging markers or time before expected disease onset. Longitudinal examinations with repeated CSF samplings are required to define when and how fast the shift to lower CSF $A \beta_{1-42}$ and higher tau levels occurs, indicating onset of amyloid deposition and neurodegeneration.

\subsection{Combined analyses of $A \beta$ and tau biomarkers}

Combining $A \beta_{1-42}$ with tau offers good discriminative value for $\mathrm{AD}$ patients compared to age-matched healthy controls, with a sensitivity of $85 \%$ and a specificity of $86 \%$. Nevertheless, when these ratios are employed to discriminate $A D$ from other dementias, a lower degree of specificity is achieved [87]. Other examinations have used the tau $x A \beta_{1-40} / A \beta_{1-42}$ ratio - referred to as the $\mathrm{AD}$ index - showing sensitivity $69 \%$ and specificity $88 \%$ [88] or the combination among $A \beta_{1-42}, A \beta_{1-38}$, and tau to make a diagnosis of $A D$ [89]. In the latter analysis, increased p-tau and the ratio $A \beta_{1-42} / A \beta_{1-38}$ account for accuracies higher than 80 and $85 \%$, respectively, to differentiate $A D$ versus non-Alzheimer dementias (NAD). The combination of p-tau with $A \beta_{1-42} / A \beta_{1-38}$ leads to a sensitivity of $94 \%$ to identify $\mathrm{AD}$ and $85 \%$ specificity to exclude NAD. The ratio $A \beta_{1-42} / A \beta_{1-38} / p$-tau, robustly distinguishing $A D$ versus $\mathrm{NAD}$, is believed to satisfy the accuracy requirements for an appropriate screening and differential diagnostic AD biomarker [89]. When reviewing this type of sensitivity and specificity figures for the AD CSF biomarkers, it should be noted that these figures come from studies based on clinically diagnosed patients, which means that a biomarker can never show a better performance than the clinical diagnosis in such studies.

\subsubsection{Progression from cognitively normal subjects to $M C I$}

The increased ratio of tau $/ A \beta_{1-42}$ and $p$-tau/A $\beta_{1-42}$ in normal subjects has been related to an amplified risk of conversion to $\mathrm{MCI}$. A study has demonstrated that about $70 \%$ of those with a high ratio versus only $10 \%$ of those with a normal ratio change to $\mathrm{MCl}$ over a $3-$ year period [90]. Later, it has been observed that all subjects who have converted to $\mathrm{MCI}$ display increased tau/A $\beta_{1-42}$ ratios (over a follow-up of 42 months), while no conversions take place in the normal ratio group [91]. In light of this, the subset of normal elderly with high ratios seems to have already developed amyloid deposition and neurodegeneration. This might denote a subgroup with a diagnosis of preclinical AD.

\subsubsection{Progression from $M C I$ to $A D$}

Numerous studies assessing the efficacy of CSF markers in predicting the risk of progression from $\mathrm{MCI}$ to $\mathrm{AD}$ indicate that diminished $A \beta_{1-42}$ and elevated t-tau and p-tau show in $\mathrm{MCI}$ a sensitivity equivalent to that observed in more advanced $A D$ [92]. Lower CSF $A \beta_{1-42} / A \beta_{1-40}$ ratios suggest risk of progression to $A D$ in subjects with very mild dementia [92]. A large longitudinal study of MCI subjects ( 18 months follow-up) has allowed the detection of a grown tau/A $\beta_{1-42}$ ratio in $90 \%$ of $\mathrm{MCI}$ subjects who have later converted to AD compared to $10 \%$ of those who have not converted [93]. Combining tau with the $A \beta_{1-42} / p-t_{10 u}{ }_{181}$ ratio has significantly predicted progression of $\mathrm{MCI}$ into more advanced $\mathrm{AD}$ in another longitudinal study (average follow-up: 4-6 years) [76].

As emphasized by Blennow et al. (2012) [94], given that the diagnostic accuracy for the combination of CSF $A \beta_{1-42}$, t-tau, and p-tau has been reported to be higher than for any biomarker alone $[76,93,95,96]$, a multiparameter assay, utilizing the Luminex ${ }^{\mathrm{TM}}$ XMAP technology (Luminex Corporation, Austin, TX, USA) to enable simultaneous quantification of these CSF biomarkers, has been developed [97]. The employment of this assay in multicenter studies on CSF biomarkers has yielded a good diagnostic performance $[75,76,79,98]$.

\subsection{Time course of $A D$ biomarkers}

Great consideration has been given to the hypothetical model for the sequence of pathologic events in AD suggested by Jack et al. (2010) according to which biomarkers reflecting $A \beta$ pathology become positive before those reproducing neuronal degeneration and tangle development [99]. Two recent examinations have addressed this issue in detail. Both studies, after scrutinizing MCI cohorts with long clinical follow-up, have identified an evident reduction in CSF $A \beta_{1-42}$ along with grown levels of t-tau and p-tau. In particular, one study has demonstrated that MCI subjects with prodromal $A D$ present with low CSF $A \beta_{1-42}$, regardless of time to dementia, whereas t-tau and p-tau are highest in patients with shorter time to conversion, thus indicating that $A \beta_{1-42}$ is completely altered before t-tau or p-tau [100]. These data support the hypothesis that modified $A \beta$ metabolism precedes tau-related disease and neuronal degeneration. The other study has disclosed that MCI subjects with elevated concentrations of injury markers namely, t-tau and p-tau - may develop faster, therefore presenting shorter time to conversion [101]. Since both analyses are crosssectional in regards to the biomarker data, the use of multiple longitudinal CSF specimens is necessary to detect the time point at which CSF biomarkers convert from physiologic to pathologic values.

\subsection{CSF biomarkers variability}

Substantial interlaboratory discrepancies, with reference to CSF biomarker levels, make assessments and comparisons of data from different laboratories problematic. As a result, globally recognized 
reference and cut-off values have not been established. For this reason, standardization efforts have been introduced to harmonize laboratory practices [102], define procedures on CSF collection and handling [103], create reference materials for assay calibration [104], and delineate reference measurement protocols [105]. In particular, the establishment of certified reference materials is presently executed as a concerted effort among the Alzheimer's Association, the International Federation of Clinical Chemistry and Laboratory Medicine, and the Institute for Reference Materials \& Measurements [106].

A universal quality-control program to evaluate total analytical variability of the best-established CSF biomarkers - A $\beta_{1-42}$, t-tau, and p-tau - has been recently initiated by the Alzheimer's Association. The aim is the standardization of CSF biomarker measurements between research and clinical laboratories to increase the analytical precision and improve the longitudinal stability of biomarker measurements [79].

To date, the major cause of the experimental variability for CSF biomarkers is due to between-laboratory factors [107]. Since global biomarker cut-off levels cannot be defined owing to the high extent of variability, each laboratory should employ internally validated cut-off values and guarantee longitudinal stability in its measurements. Progresses in standardization of laboratory protocols in conjunction with the enhancement of kit performance and the use of fully automated tools are expected to improve the effectiveness of CSF AD biomarkers for both researchers and clinicians [107].

\subsection{Upcoming candidate biomarkers}

The composition of CSF is subject to fluctuations that mirror the complexity of AD pathophysiology, involving SPs deposition, NFTs formation, gliosis/neuroinflammation, and synaptic and neuronal loss. Accordingly, lots of molecules have been proposed as potential AD biomarkers in the CSF [108]. As the power and the complexity of the "omics" disciplines such as proteomics and metabolomics - that promise to revolutionize biomedicine - has greatly advanced over the last decade, proteins encompass the majority of viable candidates. In this context, both hypothesisdriven strategies - allowing the study of definite molecules participating to $A \beta$ metabolism, neurodegeneration, neuroinflammation, and oxidative stress - and unbiased as well as targeted multianalyte profiling approaches - for instance, proteomic screening and molecular arrays - have been employed (see Fagan and Perrin (2012) for an exhaustive review on novel candidate CSF biomarkers [108]). Individually, various candidates are useful to evaluate statistical differences between cohorts of $\mathrm{AD}$ and control samples, and many of these also have the potential to increase the diagnostic accuracy of the "core" biomarkers $A \beta_{1-42}$, t-tau, and ptau. Certain promising molecules seem to be of help for diagnosis/ differential diagnosis, prognosis, and therapeutic monitoring ("theragnosis") [108].

Notably, in the framework of the "omics" revolution, mounting evidence is emphasizing the role of metabolomics to determine diagnostic biomarkers for AD. The metabolome designates a set of small-molecule metabolites discovered within a biological sample in a specific physiologic or developmental condition. Thus, different disease states disturbing biochemical networks will lead to dissimilar metabolic signatures [109]. This groundbreaking approach recognizes metabolic disturbances by assessing the activity of various metabolites at the same time. The discovery of uncommon disruptions in the metabolic network could serve to better elucidate the pathological mechanisms [110]. Notably, two analyses have reported alterations in CSF metabolome of AD. One study has showed the increase of the concentrations of eight amino acids in AD versus MCI [111]. Another larger examination, after measuring 343 analytes has also led to detect eight molecules with statistical significance; interestingly, one of these markers, cortisol, correlated with the advancement of the disease [112]. A disadvantage with metabolomics as compared with proteomics and peptidomics may be that, in contrast to several proteins, there is no data showing an established role for small (non-protein) molecules in $\mathrm{AD}$ pathogenesis.

\section{Blood prospective candidate biomarkers}

The attention on blood-based biomarkers for the diagnosis of $\mathrm{AD}$ has rapidly developed during the past decade. Although conventional AD biomarkers from CSF are highly accurate, barriers to their clinical application are still present. Since blood is a biofluid much more easily reached and manageable than CSF, searching for consistent blood-borne biomarkers is needed. In this connection, the Blood-Based Biomarker Interest Group (BBBIG), an international working group of leading $A D$ scientists from academia and industry, has been established to scrutinize the present scenario and to support the progress in the field (see Henriksen et al. (2013) for a critical perspective on the status of blood-based biomarkers for AD [13]).

Although the association of plasma $A \beta_{1-40}$ and $A \beta_{1-42}$ concentrations with incipient $A D$ has been repeatedly investigated, definite data are still lacking. Increased $A \beta_{1-40}$ or $A \beta_{1-42}$ levels have been shown to predict the development of AD [113,114]; however, other analyses have revealed no associations $[115,116]$ or opposite [117] results. A low $A \beta_{1-42} / A \beta_{1-40}$ ratio is assumed to predict future $A D[113,118,119]$. On the other side, an increased ratio $[114,120]$ or no major difference [115] in subjects with incipient $A D$, compared with those that have not developed $A D$, have been described. A recent meta-analysis has suggested that a low $A \beta_{1-42} / A \beta_{1-40}$ ratio could predict the progression of $A D$, but no such association has been observed for the single peptides [121]

With reference to tau, some studies have highlighted differences in the modulation of CSF tau levels as compared to blood. In case of hypoxic brain damage subsequent to cardiac arrest, tau is promptly released into the bloodstream but efficiently cleared, within $24 \mathrm{~h}$, in patients showing positive neurological outcome [122]. In contrast, CSF tau levels remain elevated for several weeks after an acute neurological insult [123]. In addition, tau concentrations are significantly increased in CSF of AD patients, but less in the equivalent plasma samples. Actually, measurements of tau in CSF and plasma compartments are not associated [124]. More recently, the association of plasma tau concentrations with $\mathrm{AD}$ has been appraised in a cross-sectional study including AD patients, MCI subjects, and cognitively healthy controls, using a newly developed ultra-sensitive immunoassay for the quantification of tau protein [125]. Plasma concentrations of tau appear increased in $\mathrm{AD}$ in relation to $\mathrm{MCI}$ and healthy controls. MCI-AD subjects (i.e. $\mathrm{MCI}$ converters to $\mathrm{AD}$ ) display tau levels comparable to those detected in $\mathrm{MCI}$-stable (i.e. $\mathrm{MCI}$ non-converters to $\mathrm{AD}$ ) and healthy subjects. This overlap among ranges is believed to diminish the efficacy of plasma tau as diagnostic test [125].

During the last decade, the development of mass spectrometrybased technologies has elected proteomics as the chief platform to inspect the plasma/serum proteome for the discovery of next generation biomarkers showing diagnostic, prognostic, or therapeutic efficacy [126]. Mass spectrometry-based methods, together with innovative tools in progress, are welcomed because they will significantly improve the ability to detect blood markers [127,128]. By simultaneously quantifying the levels of many plasma analytes, biomarker patterns successfully distinguishing AD patients from controls [129] or associated with $\mathrm{MCI}$ or AD have been disclosed [130]. Since the activities of most molecules are connected to immune regulation and inflammatory pathways, the existence of 
an inflammatory process in $\mathrm{AD}$ has been firmly proposed $[131,132]$. Nevertheless, such protein panels have been hard to reproduce in independent studies [133].

However, two analyses, utilizing large and well-characterized clinical cohorts, have discovered that a set of inflammatory molecules display modified expression as a function of $\mathrm{AD}$ [130,134]. Moreover, both Doecke et al. (2012) [134] and O’Bryant et al. (2011) [135] have found diagnostic accuracy across cohorts employing biomarker algorithms/profiles. These encouraging results provide additional support for the blood-based profiles/ signatures.

The discovery of plasma/serum biomarkers for a CNS disease as $\mathrm{AD}$ meets both conceptual and practical challenges [136]. No findings of transcripts/proteins/metabolites in blood have been successfully replicated to be definitively approved as AD biomarkers. Moreover, based on the data from the literature, it seems to be doubtful that a blood biomarker alone will be in itself adequate for the diagnosis of $\mathrm{AD}$. In contrast, it seems most likely to have a combination of markers: several proteins coupled with other blood-based or non-blood-based markers, as imaging [127]. It is also uncertain the existence of only one set of biomarkers for all conceivable uses in $\mathrm{AD}$. It is probable that there will be a group of biomarkers to support AD diagnosis, a different set of molecular markers to predict outcome in AD patients or conversion in $\mathrm{MCI}$, and, probably, another cluster to allow monitoring the evolution of the disease [128].

It should be also emphasized the presence of many issues in plasma/serum proteomics, including the existence of pre-analytical and analytical variables. Consequently, there is an urgent need for standardization of specimen collection/processing, quantitation, and setting strategies for managing biomarkers after their detection [137]. Progress also relies on the establishment of Standard Operating Procedures (SOPs) for the appropriate selection of patients and specimens, thus decreasing the complexity of samples to be analyzed [138]. In this regard, the Human Plasma Proteome Project (HPPP) (see http://www.hupo.org/initiatives/ plasma-proteome-project/) is an initiative conceived and launched by the Human Proteome Organization (HUPO) (available at http:// www.hupo.org/) to solve matters related to pre-analytical variability and to make attempts to establish SOPs [139]. In addition, the development of informatic tools for data management and collaborations with other disease-related initiatives of the HUPO to extend the area of plasma/serum biomarker discovery should be encouraged [140].

\section{Neuroimaging markers}

\subsection{Structural MRI markers}

Reduction of hippocampus volume, derived from structural MRI, is one of the key biomarkers of AD in the IWG [23] and NIA-AA criteria [30]. This reflects the consistent findings of reduced hippocampus volumes in $\mathrm{AD}$ and $\mathrm{MCI}$ subjects across a wide range of mono- and multicenter studies (for meta-analysis, see Clerx et al. (2012) [141]). Hippocampus volume has also been used as a secondary endpoint in several clinical trials on potential disease modifiers in AD or $\mathrm{MCI}$, including vaccination [142], muscarinic receptor agonists [143], and glutamate modulators [144]. Although widely used since more than 20 years, standardization of manual hippocampus volumetry has only begun in 2011 with the European Alzheimer's Disease Centers-Alzheimer's Disease Neuroimaging Initiative (EADC-ADNI) hippocampal harmonization project [145] that now provides an internationally consented protocol for manual hippocampus segmentation (available at http://www.hippocampal-protocol.net/SOPs/index.php). This protocol will serve to validate automated hippocampus volumetry methods [146]. The EMA regulatory authorities have endorsed a qualification process for the use of low hippocampus volume to help enrichment of study samples (available at http://www.ema.europa.eu/docs/en_GB/document_library/Regulatory_and_procedural_guideline/2011/10/WC500116264.pdf).

Few automated protocols have already been cleared for marketing as a medical device by the US FDA. However, hippocampal atrophy is not specific to $\mathrm{AD}$ and is found in other conditions, including fronto-temporal dementia [147] vascular dementia [148], Lewybody dementia [149], and depression [150].

Another structural marker beyond hippocampus volumetry is whole brain volume as longitudinal marker of disease progression and treatment effects. Automated algorithms such as voxel-based compression mapping [151] allow stable estimates of whole brain volume over time and across centers [152]. Whole brain volume has been used as secondary endpoint in several clinical trials $[142,153]$, but has become less attractive with the advent of regionally more specific protocols. These are based on local measures of gray matter concentration or cortical thickness at each point of the space and on high dimensional warping of brain scans into a common standard space [154] to estimate regional pattern of atrophy in data driven automated analyses. Longitudinal evaluation of these pattern of atrophy has begun to be used in phase Ila type clinical trials [155]. In addition, multivariate approaches such as machine learning with support vector machines have successfully been employed to derive patterns of brain atrophy that discriminate AD patients from healthy controls and MCI converters from MCI-stable subjects [156-158]. By highlighting specific topographical patterns of atrophy, these approaches have the potential to be useful to discriminate between different types of dementia [158]. Presently, scanner manufacturers are developing radiological expert systems based on these algorithms to help the radiologist to rate the presence or absence of $\mathrm{AD}$ from the pattern of atrophy derived from a single brain scan. It is important to note that these technical devices need to be employed in a multidisciplinary clinical setting where the diagnostic relevance of an imaging finding is put in the context of all other relevant clinical information by a clinical dementia specialist.

The attractiveness of MRI as endpoint in clinical trials is related to the assumption that regional brain volume can serve as in vivo surrogate of neuronal number. Clinico-pathological comparison studies have shown that hippocampus volume obtained antemortem accounted for at least $50 \%$ of variability in neuron numbers determined during autopsy [159]. The amount of variation explained by MRI-based hippocampus volumetry was above $90 \%$ when MRI scans was obtained postmortem [160]. Thus, hippocampus volumetry can be considered as an in vivo surrogate measure of hippocampal neuronal number. However, one should be careful to simply interpolate these findings to in vivo measures of cortical atrophy. In 27 antemortem cognitively intact subjects, cortical thinning determined postmortem across age cohorts was not associated with regional neuron numbers and density, but was suggested to reflect changes in neuronal and dendritic architecture [161]. Therefore, future studies need to address more specifically associations between regional brain atrophy and regional markers of neuronal degeneration. A platform implementing postmortem MRI in cranio can help such an approach through access to postmortem MRI data whose signal distribution is close to in vivo MRI scans [162]. Moreover, hippocampal sclerosis may occur in the absence of AD pathology and hippocampal atrophy is common in fronto-temporal lobar degeneration related to mutation of the progranulin $(G R N)$ or chromosome 9 open reading frame 72 (C9ORF72) genes [163].

An explicit framework has been proposed for a specific temporal sequence of biomarker changes during progression from 
asymptomatic AD to AD dementia [99]. A study of the Dominantly Inherited $\mathrm{AD}$ Network has provided evidence supporting such a sequence in asymptomatic mutation carriers of familial AD [85]. In these subjects, hippocampus atrophy was estimated to follow amyloid accumulation by 10 years and to precede onset of dementia by up to 15 years. Findings from familial forms cannot simply be transferred to sporadic AD. A recent study in sporadic AD interpolated 6-year follow-up data onto a timeline from asymptomatic to clinical manifest disease covering several decades [164]. This study estimated an onset of hippocampus atrophy 4 years before onset of clinical dementia, much later than in the familial cases. It is necessary to keep in mind that these data represent interpolations from cross-sectional [85] or maximum 6 years follow-up [164] data that are projected onto a timeline of disease progression. This projection, however, relies on assumptions of disease stages that shall actually be tested in the specific study. Therefore, there is some circularity in testing these models that need to be validated in further studies.

\subsubsection{Future directions: application of existing methods in a new} context

The next years will see increasing use of automated volumetry of hippocampus or regional brain atrophy pattern as secondary endpoints in clinical trials in prodromal $A D$ and $A D$ dementia stages. Regulatory authorities are seeking for biological surrogate markers for disease modification in a situation where neuropsychological endpoints require large cohorts and complex study designs to differentiate symptomatic from disease modifying effects [6]. Structural imaging markers will play a key role in this respect, because they provide stable measures over time and across scanners and are closely associated with underlying changes of neuronal integrity [159]; moreover, the functional relevance of regional atrophy for some specific cognitive impairments has been established [165]. Therefore, the use of structural imaging endpoints will help to reduce sample size in future clinical trials. Due to the wide availability of structural imaging markers, they are also being used to enrich the risk for AD in clinical samples of MCI subjects for clinical trials. In addition, structural MRI will help to enrich study samples of asymptomatic subjects with positive molecular biomarkers of AD. The presence of amyloid alone does not predict progression to cognitive decline with sufficient accuracy, as only $25 \%$ of amyloid positive cognitively healthy subjects progress to MCI or AD within 3 years [166]. Therefore, the presence of hippocampus atrophy together with amyloid positivity will help to select subjects with a high risk of conversion to $\mathrm{AD}$ or $\mathrm{MCI}$ within a timeframe that is relevant for a clinical trial.

Use of such markers in the well-controlled setting of a clinical trial will be in the interest of probands participating in such trials. However, these protocols will also be increasingly used for diagnostic purposes outside of clinical trials. If embedded into a multidisciplinary diagnostic setting and applied to symptomatic patients, the use of such protocols will probably help make full use of the anatomical information in a structural MRI scan to the benefit of the patient. The situation is different, when such measures are employed as screening instruments. Even today, private companies offer an analysis based on regional brain and hippocampus volumetry to people who pay to get a confirmation that their brain is still structurally intact. The problem with this business model is that we are far from knowing what an atrophic hippocampus or regional brain atrophy means in terms of risk for $\mathrm{AD}$ and dementia in an asymptomatic person without further clinical information. Moreover, there is no point in identifying a hypothetical risk of $\mathrm{AD}$ without offering an intervention and support scheme to an individual. There are still many issues to be resolved on how to adequately communicate the negative aspects of a screening to a client, such as the risk of false-positive findings, the lack of a treatment option, and the probable lack of clinical relevance of a true positive finding. The "litmus test" for the usefulness of an imaging marker is its application in the "intent to diagnose" population, i.e. in those patients that will be confronted with this diagnostic test in primary care. There is almost no evidence available on the usefulness of imaging markers, including MRI, to support an early diagnosis of AD outside of clinically highly selected samples. Future studies are needed to determine the efficacy of MRI to detect AD type pattern of atrophy in the presence of comorbidities that had usually been excluded in studies so far. In addition, the effect of a novel structural imaging marker of predementia $A D$ on care systems worldwide that have difficulties to provide adequate care even to patients in clinically manifest stages of disease needs to be assessed in future studies [4].

\subsubsection{Future directions: novel methods}

Novel methods will gain increasing importance to understand the neurobiological basis of cognitive decline in $\mathrm{AD}$. The wide availability of high-field MRI at 3 Tesla and the increasing availability of ultra-high field MRI at 7 Tesla render subfield measurements of the hippocampus a feasible diagnostic approach in selected samples. Pathological evidence suggests a selective vulnerability of hippocampal subfields in AD [167]. Manual methods to determine hippocampal subfields are based on the direct identification of anatomical boundaries and serve as goldstandard to assess the performance of automated methods. Using hippocampus subfields can significantly decrease the rate of false positive findings in the prediction of future conversion from $\mathrm{MCI}$ to AD using manual [168] or automated [169] measurement. Sequences at 7 Tesla provide higher spatial resolution, new contrasts and access to even finer substructures of the hippocampus [170,171], but the clinical relevance of these measures needs to be explored in future studies [172].

Neuropathological evidence suggests a selective involvement of specific subcortical areas, most notably the cholinergic nuclei of the basal forebrain $[173,174]$ and noradrenergic nuclei, in the locus coeruleus in AD. Based on mappings of subcortical nuclei from postmortem analyses, MRI scans in cranio will help to identify early changes in cholinergic and noradrenergic projecting nuclei in predementia and dementia stages of $\mathrm{AD}[175,176]$.

MRI-based detection of amyloid plaques has been successfully implemented in transgenic animals [177-180]. Further, a recent study indicates that detection is also possible in non-transgenic mouse lemur primates, in which plaques are formed naturally and are more similar to those found in humans [181]. MRI detection of plaques in humans will thus become a major topic of research in coming years. Using 7 Tesla MRI in human studies may allow in vivo detection of cortical amyloid deposition in the future, based on susceptibility related imaging [182] or direct visualization of amyloid plaques using intrinsic or extrinsic contrast agents. The validity of first findings and their relevance for early diagnosis will be explored in the coming years.

\subsection{Diffusion tensor imaging}

Diffusion Tensor Imaging (DTI) is a magnetic resonance (MR) technique that measures the random thermal motion of water molecules, i.e. Brownian motion, within tissue [183]. This modality does not require the injection of contrast material or radiation exposure and provide, non-invasively, unique information of the axonal organization of the brain, which is not feasible with standard MRI techniques. During the last decade, this technique has become a leading method in investigating white matter (WM) microarchitecture and integrity and has been widely employed in AD and MCI [184-187]. 
In a clinical context, modern MR high-field scanners (between 1.5 and 3.0 Tesla) allow rapid whole-brain assessment (4-10 min) of the apparent water diffusion tensor (DT) field using echo-planar imaging sequences. Images generated from DTI data may be qualitatively interpreted by using directionally encoded color (DEC) maps in which each color represents the axonal orientation of WM tracts. By contrast, using quantitative scalar metrics, most commonly the mean diffusivity (MD) and fractional anisotropy (FA), tissue integrity may be inferred [188]. DT-derived rotational invariants such as single eigen-values may be exploited in quantifying WM tracts integrity through region of interest (ROI), voxel- or tract-based spatial statistics approaches [185]. Furthermore, information regarding WM architecture may be quantified through deterministic and/or probabilistic tractography algorithm [189].

Normal human brain exhibits higher hindrance to water motion (diffusion) perpendicular to the long axis of WM bundles than parallel. This restriction is mostly attributed to macromolecules and cellular barriers (cell membrane) [190]. Neuronal damage, because of loss of the barriers, causes an increase in MD and a decrease FA.

Increased MD and decreased FA values have been reported in $\mathrm{AD}$ and $\mathrm{MCI}$ in parietal and temporal areas, including the hippocampal region, suggesting unspecific bundle degeneration $[191,192]$. Abnormal DT derived indices have also been demonstrated in frontal region, and specifically in the cingulum posterior, corpus callosum, fasciculus longitudinalis superior, and fasciculus uncinatus [193-195].

A recent study including both AD and MCI subjects [196] demonstrated a circumscribed increase in FA. These findings were aided by examining variations of a third tensor invariant, tensor mode [197] allowing to differentiate the type of anisotropy (planar, e.g. in regions of crossing fibers versus linear, in regions with one predominant orientation). Using this method, authors postulated a selective degeneration of only one of two crossing fibers suggesting a relative sparing of motor-related projection fibers crossing the association tracts of the fasciculus longitudinalis superior. In addition, DTI has been able to track the age-related WM degeneration in $\mathrm{AD}$ and, in agreement with the retrogenesis model (regions that mature late are more vulnerable to age- and disease-related degeneration), WM changes have been shown to appear earlier in specific areas such as temporo-parietal regions, the fasciculus longitudinalis inferior, and prefrontal regions [186,198,199].

Importantly, the reproducibility and robustness play a major role in DT data acquisition; this is a delicate point as tensor techniques employ extremely noisy echo-planar sequences, requiring strict quality control and quality assurance routines [200]. A recent meta-analysis highlights the high variability in both the anatomy of regions studied and DTI metrics [201]. Also, a recent European multicenter study, the European DTI Study in Dementia (EDSD) [202], revealed significant center-related effect in DT-derived measures.

One shortcoming of conventional DTI methods is related to the use of the simplistic model of a Gaussian propagator, which is not sufficiently accurate in regions where mixed tissue types can give rise to significant partial volume effects and/or where two or more WM fiber cross [203]. To this aim, more advanced methods such as Kurtosis Imaging [204-207], Diffusion Spectrum Imaging [208], higher-order tensor models [209], compartment models [210,211], and anomalous diffusion [212,213] have been introduced in order to augment their suitability in a clinical setting [214]. These upcoming techniques have been succesfully used in some pathologies, including $\mathrm{AD}$, to enhance information of earlier microstructural tissue alterations linked to disease progression $[215,216]$. Among these, Kurtosis Imaging seems to be the most promising developing modality in relation of its easy setup/ optimization and relatively short time acquisition in clinical MR scanners.

\subsection{Functional MRI markers}

Functional MRI (fMRI) represents an extraordinary technique that can study the neuronal activity through non-invasive means during specific cognitive states. This technique exploits the bloodoxygen-level-dependent (BOLD) contrasts in the vascular capillary network around the cerebral cortex. The regional metabolic demand, due to cortical activity (specific tasks/paradigms), determines an increase in local capillary hemodynamic and in the oxygenated/deoxygenated blood ratio. The increase of local deoxyhemoglobin concentration, because of its paramagnetic properties, generates an increase in local signal intensity. This technique has a relatively high spatial and temporal resolution and can be acquired along with structural MR images during the same scan session.

Several fMRI studies have been able to detect functional alterations prior to onset of cognitive impairment or AD-related structural neurodegeneration [217-219]. Task-based fMRI has been employed to study memory-related activation in the hippocampus and medial temporal lobe, typically reporting a decrease in hippocampal or parahippocampal activity during information encoding [220-224]. Also, several other studies have reported a decreased activation in the medial temporal lobe in $\mathrm{MCI}$ subjects [225-227].

A growing body of fMRI studies have focused on the "default mode network" (DMN), i.e. the interplay between a set cortical areas and the hippocampal memory system [228], the activity of which is thought to be reduced during memory intensive tasks to favor encoding and to be increased during retrieval [229]. Several studies have found dysfunctional modulation of encoding-related network activity in AD [227,230-233] or abnormal default mode pattern activity in $\mathrm{AD}$ and $\mathrm{MCI}$ patients using resting-state fMRI [234-238]. Interestingly, these results in MCI subjects have been correlated with a higher risk of progressing to AD-related dementia [239].

A bright future of fMRI or resting-state fMRI in AD and $\mathrm{MCI}$ might come in the area of novel pharmaceutical strategies, to date underexploited. Although drug-induced modulation of memoryrelated networks have been detected by fMRI [240], only few studies have demonstrated abnormal activation following, for example, long-term treatment with cholinesterase inhibitors in $\mathrm{MCI}$ and AD [241-244]. Therfore, additional studies are needed to test the potential role of fMRI as biomarker in clinical trials [245].

The speed of the innovation and the optimization of all these emerging modalites will be strictly related to stronger and faster MR gradients. Also, the integration of complementary information through a multimodal approach will be very useful to overcome the shortcomings of each single protocol, requiring advanced analysis tools which are able to integrate information from different protocols into the same processing pipeline. Similar approaches are likely to aid in better discrimination and staging of $\mathrm{AD}$ [8,246-248]. In this context, information from different modalities may be simultaneously combined using the support of machine learning algorithms enabling the classification of a single subject into a predefined group while dealing with any type of input features (e.g. genetic, clinical, and neuropsychological imaging data). Importantly, the classification performance is not significantly degraded if same-modality data are collected in different centers [249]. Recent results based on multimodal approaches have achieved encouraging results in discriminating $\mathrm{AD}$ and $\mathrm{MCI}$ subjects [250,251]. In the coming years, machine learning algorithms will be incorporated into scanner software to 
enhance the semi-automated detection of prodromal AD stages based on high-dimensional pattern recognition.

\subsection{Amyloid PET and fluorodeoxyglucose-PET markers}

\subsubsection{Fluorodeoxyglucose-PET}

PET imaging biomarkers represent highly valuable tools for non-invasive assessment of molecular and functional pathologies which are considered to be early phenomena in the development of AD. $\left[{ }^{18}\right.$ F]Fluorodeoxyglucose (FDG) is a well-established tracer, which allows the imaging of cerebral glucose metabolism, known to be tightly associated with neuronal function. Synaptic activity leads to an increased energy demand, which is covered by glial cells surrounding the synapse by increased glucose uptake from the blood [252]. Inversely, synaptic/neuronal dysfunction results in a decreased energy demand which is mirrored in regional metabolic decline.

Typical patterns of hypometabolism have been described in AD, including posterior parietal regions, precuneus, and also frontal cortical regions, sparing sensorimotor and visual cortex. These characteristic findings have been demonstrated to be superior to neuropsychological testing, regarding early and differential diagnosis of $\mathrm{AD}$, even when postmortem histopathological analysis of brain tissue served as a gold-standard [253-255].

Numerous studies were able to demonstrate that early abnormalities particularly in posterior cingulate/precuneus cortical regions have a high positive and negative predictive value with regard to prediction of conversion to $A D$ in the stage of $\mathrm{MCI}$ [256,257]. Even in some subjects with subjective memory impairment changes in metabolism have been observed, potentially reflecting early AD-typical pathological changes in the brain [258]. Interestingly, Reiman et al. (1996) were able to demonstrate abnormalities even in APOE \&4-positive subjects in younger age without any cognitive symptoms, underlining the high sensitivity of this method [259].

Regarding differential diagnosis, FDG-PET has demonstrated to be of great value because it allows the detection of different patterns of neurodegeneration, which are specific for various non$\mathrm{AD}$ (amyloid-negative) forms of neurodegeneration. This includes the subtypes of frontotemporal lobar degeneration (frontotemporal dementia, progressive aphasia, semantic dementia) as well as subtypes of Parkinson-plus syndromes such as multiple system atrophy, corticobasal degeneration, and progressive supranuclear palsy [260]. Most importantly, FDG-PET is also highly useful in differentiating within amyloid-positive subtypes of disease which cannot be distinguished on the basis of their amyloid PET-scan. This includes Lewy-body dementia, posterior cortical atrophy, and the logopenic variant of progressive aphasia [255,261].

A tight correlation between the level of metabolic decline with the degree of cognitive impairment has been demonstrated consistently [262], which qualifies this method for follow-up and therapy control studies [263]. This correlation can, however, be somewhat influenced by cognitive reserve effects, expressed in variable magnitude [264]. It has also been demonstrated that FDGPET is capable to capture therapy effects of cognitive as well as pharmacological intervention trials [265,266].

Regarding the plethora of data underlining the clinical value of FDG-PET for early and differential diagnosis of neurodegenerative disorders, as well as its complementary features as compared to amyloid-imaging, it can be expected that this method will remain an important biomarker in the coming years. Suitable MRprocedures such as resting-state fMRI or arterial spin labeling may generally bear the potential to provide information on neuronal dysfunction relatively similar to FDG-PET findings. However, the individual clinical value of these methods remains to be established in the future.

\subsubsection{Amyloid-PET imaging}

Today, several tracers for PET amyloid-imaging have been evaluated successfully, including clinical phase I-III studies in humans. The greatest overall number of studies has been performed with the tracer $\left[{ }^{11} \mathrm{C}\right]$ Pittsburgh-Compound-B (PiB), which can be considered as the current gold-standard [267]. More recently, several ${ }^{18} \mathrm{~F}$-labeled compounds have been evaluated which would allow more widespread application of this method. One of these compounds $\left(\left[{ }^{18} \mathrm{~F}\right]\right.$ Florbetapir/Amyvid $\left.{ }^{\mathrm{TM}}\right)$ has already been approved by the FDA and the EMA for commercial distribution and several others will follow in the near future. For a comprehensive review, see Rowe and Villemagne (2011) [268].

Consistently, in the great majority of all studies, a distinct uptake of the amyloid tracers has been observed in AD-patients throughout the cerebral cortex, including frontal, temporoparietal regions, and the precuneus. Whereas the basal ganglia are also regularly affected, sensorimotor and visual cortical regions show less uptake and the cerebellum is free of any relevant gray matter tracer accumulation. In young healthy control subjects, no gray matter binding of the amyloid tracers is observed but only nonspecific tracer uptake in the white matter has been demonstrated. In general, this white matter uptake has been described to be less pronounced for $\left[{ }^{11} \mathrm{C}\right] \mathrm{PiB}$ as compared to the ${ }^{18} \mathrm{~F}$ labeled compounds, which may somewhat decrease the sensitivity of the ${ }^{18} \mathrm{~F}$ labeled versions of amyloid tracers. The tracer ${ }^{18} \mathrm{~F}$-AZD4694 may form an exception, because it has been demonstrated to show comparably lower white matter retention [269]. The apparent differences in tracer distribution between different types of amyloid tracers have raised concerns about the comparability/ standardization of amyloid-PET results. In this context, different initiatives are underway, trying to define a common standard for quantification of different amyloid-imaging results [270]. This may be particularly important with regard to clinical studies.

In vivo versus postmortem histopathological cross-evaluation studies have been performed, in general confirming that increased cortical tracer-uptake corresponds to amyloid aggregation in the brain [271,272]. The tracers are also considered to be specific for amyloid deposition with the exception of $\left[{ }^{18} \mathrm{~F}\right]$ FFDNP, which has been demonstrated to bind also to tau aggregates [273]. Although the tracers are specific for the protein aggregation (i.e. amyloidplaques), the protein aggregation is not specific for AD. For example, it is known from histopathological studies that in Lewybody dementia, amyloid plaques aggregation will be found in the brain in addition to the pathognonomic synuclein deposits, in most cases [274]. Thus, amyloid-imaging may not be able to differentiate between Lewy-body dementia and AD. Furthermore, amyloidimaging alone may not be helpful with regard to distinguishing between amyloid-positive subtypes of $A D$ (typical $A D$, logopenic variant of progressive aphasia, and posterior cortical atrophy) [261].

With regard to early diagnosis, a number of studies demonstrated a high predictive value of a positive amyloid-scan in the stage of MCI with regard to conversion to AD [275,276]. Even in subjects with subjective memory impairment, increased levels of amyloid deposition have been described [277] and Reiman et al. (2009) were able to demonstrate elevated amyloid-levels in asymptomatic carriers of the APOE $\varepsilon 4$ allele [278]. Furthermore, in a relevant proportion of elderly subjects without any cognitive complaints elevated cortical tracer-uptake was observed consistently. The meaning of these findings is not definitely clear so far, but a number of findings indicate that these subjects may indeed suffer from early AD-pathology, potentially leading to dementia later in life. This includes relatively worse performance in cognitive tests $[279,166]$ as well as abnormal findings in other imaging tests such as resting-state connectivity [280]. In addition, recent trials in 
autosomal dominantly inherited forms of $\mathrm{AD}$ were able to demonstrate cerebral amyloid deposition decades ahead of the expected onset of disease, using amyloid-PET. However, currently the expected time to a potential conversion to AD cannot be estimated on the basis of a positive amyloid-scan alone. Furthermore, it has to be taken into account that amyloid-imaging is not suitable to detect soluble amyloid oligomers, which have been discussed to potentially represent the most toxic species [281].

Only a limited correlation has been observed between in vivo measured amyloid burden and cognitive decline. This may particularly depend on the stage of disease: (I) in cognitively healthy elderly subjects amyloid pathology may not yet have induced neurotoxic effects downstream from amyloid aggregation sufficient enough to have an impact on cognition; (II) in patients with manifest Alzheimer's dementia, a plateau of amyloid deposition has been observed, indicating that amyloid deposition reaches saturation, whereas subsequent neurodegeneration (and cognitive decline) continues [282]. As for FDG-PET, in the presence of cerebral compensation mechanisms, expressed to different degree in different subjects, it may also lead to a discrepancy between cortical amyloid load and symptomatic appearance. These factors do not necessarily limit the value of amyloid-imaging for therapy trials. First, the value of amyloid-imaging with regard to patient selection is undoubted. Second, amyloid-imaging may allow the measurement of the increase in amyloid deposition over time particularly in early stages, i.e. ahead of a plateau phase. Finally, it has been demonstrated that the response to anti-amyloid therapy may be quantified at least in a group based evaluation [283].

Regarding the commercial availability of amyloid-imaging tools, appropriateness of use criteria have recently been published in the Journal of Nuclear Medicine and Molecular Imaging [284]. These criteria suggest a useful application of amyloid-imaging in patients with $\mathrm{MCI}$, in $\mathrm{AD}$ with atypical presentation (e.g. earlyonset) and when the diagnosis is uncertain after evaluation by a dementia expert. Without doubt, amyloid-imaging may represent one of the most important biomarkers for scientific and clinical assessment of $\mathrm{AD}$ in the future. The establishment of this sophisticated method for in vivo assessment and quantification of a molecular neuropathology will certainly also depend on reimbursement issues and on the question if anti-amyloid therapy trials will be followed further and yield in first promising pharmacotherapeutic approaches.

\subsubsection{Complementary value of FDG-PET and amyloid-PET and order of abnormalities}

As mentioned above, recently introduced guidelines recommend the integration of biomarkers into the classification/ estimation of likelihood of preclinical, prodromal, and manifest stages of AD. According to these guidelines, both FDG-PET and amyloid-PET are suited to play an important role as diagnostic biomarkers in all stages of disease. In short, the proof of amyloid pathology (as possible with amyloid-PET) accompanied by proof of neuronal injury (as possible with FDG-PET) and finally proof of cognitive impairment sum up to an increasing probability for AD [25-27]. All these guidelines are based on the assumption that amyloid pathology is the first biomarker to become positive, followed by neuronal injury/tau-pathology and finally cognitive decline. Some recent data from a study in subjects with inherited $\mathrm{AD}$ in presymptomatic stages seems to confirm this notion in principle [85]. On the basis of the currently available information, it seems that amyloid-PET and $A \beta_{1-42}$-changes in the CSF behave relatively similar with regard to early detection of ongoing ADpathology. Bateman et al. (2012) were able to demonstrate a very early decline in CSF $A \beta_{1-42}$-levels in mutation carriers but coming from a higher preexisting overall $A \beta_{1-42}$-level [85]. Thus, an early detection of ongoing disease on the basis of single time-point absolute $A \beta_{1-42}$ CSF levels would not be possible. A significant difference in $A \beta_{1-42}$-levels between carriers and non-carriers was detected relatively later as compared to the onset of significant abnormalities detected with amyloid-PET.

The mentioned guidelines and recent models of biomarker time courses treat CSF tau, FDG-PET, and structural MRI as equivalent markers of neuronal injury, appearing subsequently to amyloid pathology [285]. However, this assumption may represent an oversimplification for several reasons. First, it is known that FDG mirrors neuronal dysfunction and, from a pathophysiological point of view, it appears obvious that functional changes should be detectable ahead of neuronal loss/brain atrophy. In fact, studies were able to demonstrate higher sensitivity of FDG-PET as compared to structural MRI with regard to prediction of $A D$ in the stage of MCI. FDG-PET may also be able to monitor changes in neuronal function in response to therapy, which may not be detectable with MRI or CSF tau measurements.

Furthermore, several recent findings are challenging the classic amyloid-hypothesis. This includes imaging studies demonstrating the presence of neuronal injury in absence of any proof of amyloid pathology [286,287]. Thus, further studies are required to gain deeper insights into the actual order of appearance of the pathologies and the threshold of their detectability. In this context, novel PET-tracers for tau-imaging may be of extraordinary importance. Fortunately, first successful experiments to establish such novel imaging biomarkers are currently on their way [288].

As mentioned above, the advantage of imaging biomarkers as compared to CSF biomarkers can be found in the provision of information not only on the presence of a certain pathology but also about the topography and the actual extent in the brain. This may be an important advantage with regard to disease staging, follow-up/therapy control, and differential diagnosis. Novel imaging instrumentation such as hybrid PET/MR scanners may offer an additional opportunity to merge the complementary information from different imaging modalities into new integrated in vivo biomarkers of neurodegeneration.

\section{Neuroelectrical and neuromagnetic markers}

The full potential of neurodynamic time-sensitive biomarkers using electroencephalography (EEG) [289] and magnetoencephalography (MEG) [290] for quantification of degenerative brain changes during various stages of $\mathrm{AD}$ has yet to be realized. Subtle but consistent deviations in the electromagnetic neuronal dynamics have been shown to precede explicit cognitive manifestations in AD [291] which could enable a future role of EEG/MEG biomarkers not only as a clinical diagnosis and treatment option, but also as a new mode for AD stage discovery. Dramatic progresses in dense-array active-EEG and MEG sensor technology, as well as in advanced signal processing techniques [292] have generated a recent surge of interest to use these promising capabilities in the context of improved clinical AD diagnosis. The added value of the EEG/MEG markers as an inexpensive, fast, and time-resolved tool is set to be explored rigorously both as a standalone approach and as a complementary measure together with other biomarker modalities.

\subsection{Resting-state neuroelectrical/neuromagnetic markers}

The spontaneous activity of the brain's resting-state networks (RSN), while the subject is idle with eyes closed or open, can be characterized by quantitative EEG/MEG measures (qEEG/qMEG), often using frequency band power or time-frequency estimates. Brainwave components of the resting EEG could be altered in the early stages of AD. There is evidence that EEG power in the alpha band declines with AD-related cognitive impairment [293]. Other 
studies have shown enhanced low-frequency brain oscillation in the theta [294] and delta bands in temporal and occipital areas as well as reduction of beta power in temporal and occipital areas in MCI [295]. However, frequency band-power methods need to address some current limitations, notably regarding the necessity to adjust band limits depending on task and individual, as well as to study more completely each band's significance in relation to neuronal phenomena.

A next generation of more sophisticated resting-state signal analysis approaches [292] is set to improve upon and to replace band-power markers in the next decade by capturing better the complex characteristics and dynamics of progressive neurodegeneration and aging. Promising methods involve brain connectivity [296], global synchronization, synchronization likelihood [291], detrended fluctuation analysis, approximate entropy, mutual information, source localization, and a host of further non-linear signal features. This will open new possibilities and raise new questions such as a recent study showing in AD not only that an EEG synchronization marker was suppressed in the $10-30 \mathrm{~Hz}$ range (upper-alpha and beta bands) but also that the temporal fluctuations of this synchronization measure carry additional diagnostic value in the lower alpha and beta bands [297].

\subsection{Functional neuroelectrical/neuromagnetic markers}

Functional neuroelectrical/neuromagnetic biomarkers represent an emerging candidate for a diagnostic tool in AD clinical practice, created to evaluate specific functional activities of the brain, as opposed to resting-state. Their main purpose is the dynamic detection of cognitive-task-related deviations in brain function following the onset of $\mathrm{AD}$ due to impairment of neuronal connections or neuronal components participating in the functional response. Such deviations are not always manifested during the resting-state due to the targeted activation of task-related pathways and areas of the brain. Although existing topographical and pathophysiological biomarkers have shown substantial capabilities for identification or follow-up of AD [298], functional neurodynamic measures provide differential information that is advantageous and complementary in relation to cognitive impairment and progression of the disease [299]. AD biomarkers of pathophysiological type using amyloid-imaging can expose early changes in cognitively normal individuals leading to dementia, yet the subsequent structural brain changes during the various stages of the disease are more optimally followed using topographical biomarkers such as MRI and FDG-PET [300]. There is a clear need to bridge the drawbacks of these biomarker approaches in view of the challenging tasks of detection and follow-up of subjects on the way to convert to clinical AD [301].

Currently, most functional EEG biomarkers [302] are based on spatio-temporal features such as the peak amplitude or latency of event-related potentials (ERPs) [303] (e.g. the N400/P600 ERPs which are cognitive indicators of episodic memory encoding [304]). Yet, the event-related potential/event-related field (ERP/ERF) approach is in need to address further some well-known usability issues [305]. Similarly to resting-state measures, in the future a wider application of new biomarkers based on evoked spatiospectro-temporal measures and task-related dynamic synchrony methods will be needed to bring in additional capability for handling single-trial EEG/MEG data more reliably, and to reflect the state of the functional brain networks for each individual subject.

\subsection{Future steps toward establishing the neuroelectrical/ neuromagnetic markers}

The main challenge for establishing the EEG/MEG biomarkers as an $\mathrm{AD}$ diagnostic instrument is the diversity of approaches in existing studies. While this richness of possibilities is quite promising, a first practical step would be to select a first battery of neurodynamic biomarkers based on existing results and to initiate proposals for full standardization and implementation in practice. A modular approach would ensure that future advances can be efficiently integrated. Possible standardization modules could include data recording procedures, specific guidelines on suppression of signal noise interferences, as well as recommendations on feature extraction and diagnostic decision-making. Special attention is necessary to ensure an adaptive approach as a prerequisite for success, including the integration of individual biomarker baselines for the subjects. The goal to recognize reliably each $\mathrm{AD}$ stage using EEG/MEG biomarkers is particularly challenging since it is necessary to overcome known brain plasticity effects due to compensatory mechanisms in the preclinical and prodromal stages of $\mathrm{AD}$ as neurodegenerative and cerebrovascular lesions impose progressive impairment. The final steps would involve an extensive multi-step, multi-center validation of the biomarker standards, as well as a modality integration with other measures (a compatibility study).

The existing record of neuroelectrical/neuromagnetic biomarker performance in the scientific literature suggests a promising potential in enhancing the reliability and specificity of AD prognosis while circumventing technical, experimental, financial and logistic limitations of other biomarker measurements [306].

\section{Regulatory perspectives}

Despite remarkable progress in understanding the molecular underpinnings of $\mathrm{AD}$ during the last three decades, there are no effective interventions for altering the progression of the disease. Even those few medicinal products approved for symptomatic treatment of mild to moderate stages the disease are inadequate for long term amelioration of symptoms in more severe cases. The positive results of pre-clinical studies aimed at rescuing synaptic dysfunction or preventing behavioral impairment in animal models [307] have yet to be translated into disease-modifying compounds in humans. The latest clinical trial failure of bapineuzumab and the very modest results from two major phase III studies for solanezumab raises several questions regarding: (I) prevailing ideas-theories about the pathogenesis of the disease, (II) the appropriates of the therapeutic targets, (III) selection or inclusion criteria of subjects into clinical trials, e.g. pre-clinical subjects versus mild-moderate, and (IV) study design. US and EU regulatory agencies are facing these questions as well recent recommendations of various task forces for clinical trials in AD.

Recently, the FDA has proposed a draft guideline for Industries (available at http://www.fda.gov/downloads/Drugs/GuidanceComplianceRegulatoryInformation/Guidances/UCM338287.pdf) allowing alternative targeting of intervention at the early stages of AD. According to this new guidance FDA suggests potential approaches to clinical trial design and execution that allow for regulatory flexibility and innovation [308]. There they cover the selection of patients for trials at early stages of $A D$ and for this there is a consensus within the $\mathrm{AD}$ research community that clinical diagnosis of early cognitive impairment might be coupled with specific appropriate biomarkers of disease. Diagnostic criteria have been established and are under validation by various working groups [6]. Such biomarkers include brain A $\beta$ load, as measured by PET and CSF levels of $A \beta$ and tau proteins [309] as outlined earlier in this article.

However, adequate validation of these biomarkers is still lacking despite over 19,000 published papers. Approximately 150 longitudinal studies related to the biomarkers of interest were identified which included subjects who had objective cognitive impairment but no dementia at baseline. The authors report that 
the body of evidence for these imaging and CSF biomarkers is still limited and variable across the different types of biomarkers [310]. As far as the CSF biomarkers are concerned, it was recently reported that the overall variability of data coming from a total of 84 laboratories remains too high to allow the validation of universal biomarker cut-off values for the specific intended use [107], which underscores the urgent need for better harmonization and standardization of these methods.

The use of biomarkers as endpoints in earlier stages of drug development is well established for regulators, and there are examples to approve medicinal products on the basis of their effects on validated surrogate markers, e.g. antihypertensives, or cholesterol-lowering products. However, these examples have been considered as validated surrogate markers as they allow substitution for a clinically relevant end point. In their validation a link between a treatment-induced change in the biomarker and long-term outcome of the relevant clinical measure was undoubtedly established. Therefore, the regulatory requirements on biomarkers used as endpoints in clinical trials are high as outlined earlier [309]. In consequence EU regulators help applicants in their research and development by issuing opinions on the acceptability of using such biomarkers or a distinct methodology in clinical trials. Since 2011, EMA's Committee for Medicinal Products for Human Use (CHMP) has adopted and published four of these qualification opinions for use in the development of medicines for AD. Three of these qualification opinions are for biomarkers to help identify and select patients at the pre-dementia stage of the disease. The fourth one is for a biomarker to be used to select patients for clinical trials in mild and moderate AD. In August 2013, a public consultation ended on a qualification opinion for a novel model of disease progression and trial evaluation in mild and moderate AD. The simulation tool is intended to provide a quantitative rationale for the selection of study design and inclusion criteria for the recruitment of patients.

In the diagnostic area, the approval of the first radiopharmaceutical for PET imaging of $A \beta$ neuritic plaques in the brain by the European Commission, in January 2013, on the recommendation of the CHMP has been another step forward. This diagnostic agent can be used in patients who are being evaluated for AD and other causes of cognitive decline. Two other diagnostic radiopharmaceuticals for $\mathrm{AD}\left(\left[{ }^{18} \mathrm{~F}\right]\right.$ Florbetaben and $\left[{ }^{18} \mathrm{~F}\right]$ Flutemetamol) are currently under evaluation by the CHMP. However, interpretation of amyloid scans is not without hurdles: amyloid positivity does not reliably distinguish between clinical diagnoses, so that neuropsychiatric normal people as well as those with $\mathrm{MCI}, \mathrm{AD}$, and other neurodegenerative diseases can all be "amyloid positive". Therefore, a positive amyloid scan must be considered in the full clinical picture of a patient, on the other hand a negative amyloid scan indicates that the likelihood of cognitive impairment due to $A D$ is low [311,312].

Another issue in future clinical trials is the appropriate choice of clinical endpoints. In established AD the CHMP guidance requires co-primary endpoints in cognition (mandatory) together with functional or global outcome measures; moving now to earlier asymptomatic or prodromal stages of $\mathrm{AD}$ might change this requirement. Thus, the FDA suggests for clinical trials focusing on patients in whom overt dementia seems imminent the use of a single scale that combines assessment of both cognition and function such as the score on the Clinical Dementia Rating Sum of Boxes (CDR-SB) [308]. For patients whose disease is at an even earlier clinical stage, it might be possible to approve a drug through an accelerated procedure pathway on the basis of assessment of only cognitive symptoms in the US. The accelerated approval mechanisms will allow drugs that address an unmet medical need to be approved on the basis of a surrogate endpoint or an intermediate clinical endpoint (i.e. a sensitive cognitive measure). In the EU, a similar approach is possible via a "conditional" approval, which implies that the applicant accepts after such an preliminary approval the obligation to carry out further long-term clinical studies to confirm clinical efficacy and safety. Only after the approval and long-term treatment, it would be possible to properly follow the amelioration of cognitive and behavioral disorders as well as the slowing of the progression of neurodegenerative lesions as shown by neuroimaging techniques [309]. Pharmaceutical industry is encouraged to seek scientific advice on their development program as soon as possible with the regulators, if they intend to use new methods to define the patient population or specific study designs and assessment tools. For instance, Richard et al. (2013) have proposed a new memory test for improving the diagnostic accuracy in patients with mild cognitive impairment recently. In particular, the Net Reclassification Improvement (NRI), followed by MRI and CSF analysis, might be an attractive and easy way to interpret certain measures for clinicians [313]. The development and validation of such new assessment tools is encouraged by regulators.

By the end of 2013, CHMP will decide whether or not there is a need to revise the guideline on the clinical investigation of medicines for the treatment of $\mathrm{AD}$ on the basis of new knowledge obtained from the use of biomarkers in clinical evaluation and new trends in research and development. It has already been acknowledged that AD is more a "continuum" of different stages and that the focus of new drug development has shifted to earlier stages. It is desirable that regulators and all involved stakeholders work together to decide the best design at the various stages of disease of the new clinical trials for AD prevention and treatment.

\section{Conclusions}

According to the new diagnostic criteria of $A D$ recommended by the IWG [21,23] and the revised NIA-AA [26-28] initiatives, biomarkers are expected to play a prominent role in future development-validation of technologies-algorithms for: (I) accurate detection of people in the early stage of the disease, (II) more reliable diagnosis, and (III) accurate prognosis or prediction of asymptomatic people at elevated risk. This will be also possible in equivocal cases with unusually presenting clinical symptoms and problematic classification/differential diagnosis [314]. As argued by Visser et al. (2012), the IWG and the NIA-AA criteria display both commonalities and important differences [20]. Notably, they concur in recognizing the onset of AD prior to dementia [24] and highlight the employment of biomarkers as critical and supportive data for the early diagnosis of prodromal AD. In clinical trials, biomarkers can be utilized to enrich early or asymptomatic AD, thus decreasing both the extent of heterogeneity within diagnostic groups and the number of individuals necessary to detect statistically significant group differences. As a result, the statistical power will be increased [315].

Besides their diagnostic significance, biomarkers may contribute to the progress in the development of novel drugs for the treatment of AD related molecular mechanisms. They may be employed for the in vitro monitoring of drug discovery plans intended to identify new molecules inhibiting amyloidogenic mechanisms and to provide surrogate measures assessing treatment efficacy of novel $A \beta$-targeting drugs, which would decrease the time and cut the costs of clinical trials [94]. In addition, biomarkers may help demonstrate the usefulness of a certain therapy in a specific patient, thus assisting the physician to find the proper medication. Intriguingly, Lu et al. (2013), employing solid-state nuclear magnetic resonance (NMR) approach, have reported the existence of an original structural model of $A \beta$ fibrils 
from AD brain, characterized by significant differences from in vitro fibrils [316]. These novel structural data can be utilized to construct novel structure-specific PET radioligands for in vivo amyloid-imaging and conceptualize more selective small molecule inhibitors, and therapeutic antibodies [317]. These unique structure-specific PET radioligands, once validated by future follow-up studies, might be used in cooperation with CSF and blood biomarkers to help refining patient stratification [317].

Controlled and observational longitudinal studies utilizing combinations of biofluid markers in conjunction with other types of diagnostic and therapeutic approaches are required. In the absence of such studies, it is challenging to recommend exhaustive diagnostic algorithms that integrate fluid biomarkers. Moreover, the paucity of standardized procedures to quantify the existing biomarkers impedes the use of validated biomarker cut-off values to guide and monitor clinical decision-making. Attaining the validation of these cut-off points is one of the key objectives of present research performed into biomarker discovery both for $\mathrm{AD}$ and for other neurological disorders [318].

Finally, the standardization of the methodologies and the development of external control assays/tools/methodologies are compulsory requirements to enable the successful use of biomarkers in the diagnosis and management of AD [6,319].

At present, trials aiming at exploring early $\mathrm{AD}$ have been developed. In this regard, an umbrella group-the Collaboration for Alzheimer's Prevention (CAP), sponsored by Fidelity Biosciences Research, Inc., and the Alzheimer's Association - has been established which incorporates three separate, but interconnected, long-term prevention initiatives [18]: the Dominantly Inherited Alzheimer's Network (DIAN) [85], the Alzheimer's Prevention Initiative (API) [320], and the Anti-amyloid Treatment of Asymptomatic Alzheimer's (A4) trial [321]. CAP has been promoted to harmonize the studies and encourage data sharing: it exists as a setting for DIAN, API, and A4 to keep a systematic discussion among them as they plan and execute their preclinical treatment trials [18]. All of the three trials will focus on the concept that AD pathological mechanisms initiate long before the onset and progress of dementia and that amyloid is critically involved in the disease pathogenesis [322].

The paradigm shift toward early AD detection/characterization/ diagnosis is essential to redefine and launch successful interventional trials. Such a paradigm embraces both secondary prevention (i.e. preventing the progression of pathological mechanisms and subsequent symptoms) and primary prevention (i.e. preventing the beginning of molecular and cellular mechanisms/signaling pathways). This objective may be attained by integrating the clinical trial approach to disease into a public health model, using long-term longitudinal databases that include large populations [323]. In this connection, significant initiatives showing a worldwide perspective are: the Organization for Economic Cooperation and Development (OECD) Task Force on AD (available at http://www.oecd.org/), the EU/US Task Force on Clinical Trial Development in AD [18,324], and the non-profit corporation Prevent Alzheimer's Disease 2020 (PAD2020) (available at http:// www.pad2020.org) [17], all stressing that a world-wide database should be established by integrating/expanding existing cohorts and registries [323].

Given the vibrant and as of yet relatively unexploited future potential of the multimodal biomarker development, the current status of the integration of biomarkers in clinical trials seems only the beginning of the evolving paradigmatic "systems biology and neural network" era of AD [7,12]. This seems to be the most promising road ahead to breakthrough advances in this highly complex scientific arena. It is recognized that we can learn much from existing research in early asymptomatic populations as well as in familial autosomal dominant AD. However, it will be necessary to chart the full spectrum biomarker map in complex, non-linear sporadic AD [7,12] to progress and improve effective treatment perspectives.

Systems biology is an emerging interdisciplinary approach to $A D$ research [12] that allows the integrated examination and assessment of interrelated biological pathways where structurally/functionally different biomolecules are simultaneously measured over time in cells, networks of cells, organs, or whole organisms [325]. Systems biology, embracing a large set of divergent methodological approaches, has become realistic owing to multiple high-throughput "omics" technologies, namely genomics/epigenomics, transcriptomics, proteomics, and metabolomics/lipidomics. These platforms, in association with accurate bioinformatic analyses using powerful computational and statistical modeling tools, will permit the investigation of various types of molecular interactions [325], such as transcriptional modules [326], gene-interaction networks [326], protein-protein interaction networks [327], and signaling networks [327]. Studying these network models will help unveil previously unknown molecular network properties of AD as well as identify genes, proteins, and cellular pathways critically involved in $\mathrm{AD}$ mechanisms. This, in turn, will be of support for the detection of the most appropriate gene and protein targets for AD treatment.

\section{Conflict of interest}

H.H. declares no competing financial interests related to the present article. During the last two years he has received lecture honoraria and/or research grants and/or travel funding and/or participated in scientific advisory boards and/or as a consultant to diagnostic, biotechnology, and pharmaceutical companies involved in the manufacture and marketing of biomarkers and/or diagnostics and/or drugs or medicinal products for cognitive impairment and Alzheimer's disease including Boehringer-Ingelheim, Bristol-Myers Squibb, Elan Corporation, Wyeth, Novartis, Eisai Inc., Pfizer, Schwabe, Sanofi-Aventis, Roche Pharmaceuticals and Diagnostics, GE Healthcare, Astra-Zeneca, Avid, Eli Lilly and Company, Janssen-Cilag, Merz Pharmaceuticals, GlaxoSmithKlineBiologicals, Jung-Diagnostics, Thermo Fisher Scientific Clinical Diagnostics, Cytox. He is co-inventor in pending patent submissions relating to biological markers and/or diagnostics and has not received any royalties.

K.B. has served at Advisory Boards for Innogenetics, Kyowa Hakko Kirin Pharma, Pfizer, and Roche.

H.B. declares no conflicts of interest. He does not have any financial support from commercial companies regarding this study.

A.D. declares the following potential conflicts of interest: Piramal, AVID Pharmaceuticals/Lilly, GE Healthcare, and Siemens Healthcare.

O.C. declares no financial activities related to the present article. He reports having received lecture fees from Lundbeck, consulting fees from Guerbet and no other biomedical financial interests or any potential conflicts of interest.

A.B. received honoraria from the Wolfson Foundation for reviewing the scientific project, and from Ludbeck for giving a talk as a speaker. He also received research support for the French Agency for Research.

P.S.A. declare no competing financial interests related to the present article. He serves on a scientific advisory board for NeuroPhage; he has served as a consultant to Elan Corporation, Wyeth, Eisai Inc., Schering-Plough Corp., Bristol-Myers Squibb, Eli Lilly and Company, NeuroPhage, Merck \& Co., Roche, Amgen, Genentech, Inc., Abbott, Pfizer Inc, Novartis, Bayer, Astellas, Dainippon, Biomarin, Solvay, Otsuka, Daiichi, AstraZeneca, Janssen, 
Medivation, Ichor, Toyama, Lundbeck, Biogen Idec, iPerian, Probiodrug, Somaxon, Biotie, Cardeus, Anavex, Kyowa Hakko Kirin Pharma, and Medtronic.

B.D. has participated to Advisory Boards for Amivid, Eli Lilly, and Roche. His Institution has received grants from Roche and Pfizer. S.L., S.J.T., F.G., R.N., H.Z., L.B., C.D., S.E., K.Br., S.Le., and Z.S.K. declare no competing financial interests.

\section{Acknowledgements}

The authors would like to thank the FRA, Fondation Pour La Recherche Sur Alzheimer, Paris, France.

H.H. was supported by the Katharina-Hardt-Foundation, Bad Homburg vor der Höhe, Germany.

This work was supported by the program "Investissements d'avenir" (Grant Number ANR-10-IAIHU-06). The work of O.C. is supported in part by ANR (project HM-TC, Grant Number ANR-09EMER-006) and by France Alzheimer Association (project IRMA7).

P.S.A. receives research support from Eli Lilly and Company and Baxter International Inc., and the NIH [NIA U01-AG10483 (PI), NIA U01-AG024904 (Coordinating Center Director), NIA R01AG030048 (PI), and R01-AG16381 (Co-I)].

\section{References}

[1] Braak H, Braak E. Neuropathological staging of Alzheimer-related changes. Acta Neuropathol 1991;82:239-59.

[2] Thal DR, Capetillo-Zarate E, Del Tredici K, Braak H. The development of amyloid beta protein deposits in the aged brain. Sci Aging Knowledge Environ 2006;2006:re1.

[3] Hyman BT, Phelps CH, Beach TG, Bigio EH, Cairns NJ, Carrillo MC, et al. National Institute on Aging-Alzheimer's Association guidelines for the neuropathologic assessment of Alzheimer's disease. Alzheimers Dement 2012;8:1-13.

[4] Hampel H, Prvulovic D, Teipel S, Jessen F, Luckhaus C, Frölich L, et al. The future of Alzheimer's disease: the next 10 years. Prog Neurobio 2011;95:718-28.

[5] Blennow K, Hampel H, Weiner M, Zetterberg H. Cerebrospinal fluid and plasma biomarkers in Alzheimer disease. Nat Rev Neurol 2010;6:131-44.

[6] Hampel H, Frank R, Broich K, Teipel SJ, Katz RG, Hardy J, et al. Biomarkers for Alzheimer's disease: academic, industry and regulatory perspectives. Nat Rev Drug Discov 2010;9:560-74.

[7] Hampel H, Lista S, Khachaturian ZS. Development of biomarkers to chart all Alzheimer's disease stages: the royal road to cutting the therapeutic Gordian Knot. Alzheimers Dement 2012;8:312-36.

[8] Ewers M, Sperling RA, Klunk WE, Weiner MW, Hampel H. Neuroimaging markers for the prediction and early diagnosis of Alzheimer's disease dementia. Trends Neurosci 2011:34:430-42.

[9] Trojanowski JQ, Hampel H. Neurodegenerative disease biomarkers: guideposts for disease prevention through early diagnosis and intervention. Prog Neurobiol 2011;95:491-5.

[10] Nicotera P, Hampel H. Perspectives of worldwide translational biomarke research in neurodegenerative diseases. Prog Neurobiol 2011;95:496-7.

[11] Bertram L, Hampel $\mathrm{H}$. The role of genetics for biomarker development in neurodegeneration. Prog Neurobiol 2011;95:501-4.

[12] Hampel H, Lista S. Alzheimer disease: from inherited to sporadic AD-crossing the biomarker bridge. Nat Rev Neurol 2012;8:598-600.

[13] Henriksen K, O’Bryant SE, Hampel H, Trojanowski JQ, Montine TJ, Jeromin A, et al. The future of blood-based biomarkers for Alzheimer's disease. Alzheimers Dement 2013. http://dx.doi.org/10.1016/j.jalz.2013.01.013. pii: S15525260(13)00045-9.

[14] Weiner MW, Veitch DP, Aisen PS, Beckett LA, Cairns NJ, Green RC, et al. The Alzheimer's Disease Neuroimaging Initiative: a review of papers published since its inception. Alzheimers Dement 2012;8:S1-68.

[15] Frisoni GB. Alzheimer's disease neuroimaging initiative in Europe. Alzheimers Dement 2010;6:280-5.

[16] Carrillo MC, Bain LJ, Frisoni GB, Weiner MW. Worldwide Alzheimer's disease neuroimaging initiative. Alzheimers Dement 2012;8:337-42.

[17] Hampel H, Lista S. Use of biomarkers and imaging to assess pathophysiology, mechanisms of action and target engagement. J Nutr Health Aging 2013;17:54-63.

[18] Vellas B, Carrillo MC, Sampaio C, Brashear HR, Siemers E, Hampel H, et al. Designing drug trials for Alzheimer's disease: what we have learned from the release of the phase III antibody trials: a report from the EU/US/CTAD Task Force. Alzheimers Dement 2013;9:438-44

[19] Blennow K. Biomarkers in Alzheimer's disease drug development. Nat Med 2010;16:1218-22.
[20] Visser PJ, Vos S, van Rossum I, Scheltens P. Comparison of International Working Group criteria and National Institute on Aging-Alzheimer's Association criteria for Alzheimer's disease. Alzheimers Dement 2012;8: 560-3.

[21] Dubois B, Feldman HH, Jacova C, Dekosky ST, Barberger-Gateau P, Cummings $\mathrm{J}$, et al. Research criteria for the diagnosis of Alzheimer's disease: revising the NINCDS-ADRDA criteria. Lancet Neurol 2007;6:734-46.

[22] Cummings JL, Dubois B, Molinuevo JL, Scheltens P. International Work Group criteria for the diagnosis of Alzheimer disease. Med Clin North Am 2013;97:363-8

[23] Dubois B, Feldman HH, Jacova C, Cummings JL, Dekosky ST, Barberger-Gateau $P$, et al. Revising the definition of Alzheimer's disease: a new lexicon. Lancet Neurol 2010;9:1118-27.

[24] Cummings J. Alzheimer's disease diagnostic criteria: practical applications. Alzheimers Res Ther 2012;4:35

[25] Isaac M, Vamvakas S, Abadie E, Jonsson B, Gispen C, Pani L. Qualification opinion of novel methodologies in the predementia stage of Alzheimer's disease: cerebrospinal fluid related biomarkers for drug affecting amyloid burden-regulatory considerations by European Medicines Agency focusing in improving benefit/risks in regulatory trials. Eur Neuropsychopharmacol $2011 ; 21: 781-8$

[26] Sperling RA, Aisen PS, Beckett LA, Bennett DA, Craft S, Fagan AM, et al. Toward defining the preclinical stages of Alzheimer's disease: recommendations from the National Institute on Aging-Alzheimer's Association workgroups on diagnostic guidelines for Alzheimer's disease. Alzheimers Dement 2011;7:280-92.

[27] Albert MS, DeKosky ST, Dickson D, Dubois B, Feldman HH, Fox NC, et al. The diagnosis of mild cognitive impairment due to Alzheimer's disease: recommendations from the National Institute on Aging-Alzheimer's Association workgroups on diagnostic guidelines for Alzheimer's disease. Alzheimers Dement 2011;7:270-9.

[28] McKhann GM, Knopman DS, Chertkow H, Hyman BT, Jack Jr CR, Kawas CH, et al. The diagnosis of dementia due to Alzheimer's disease: recommendations from the National Institute on Aging-Alzheimer's Association workgroups on diagnostic guidelines for Alzheimer's disease. Alzheimers Dement 2011;7:263-9.

[29] Jonsson T, Atwal JK, Steinberg S, Snaedal J, Jonsson PV, Bjornsson S, et al. A mutation in APP protects against Alzheimer's disease and age-related cognitive decline. Nature 2012;488:96-9.

[30] Jack Jr CR, Albert MS, Knopman DS, McKhann GM, Sperling RA, Carrillo MC, et al. Introduction to the recommendations from the National Institute on Aging-Alzheimer's Association workgroups on diagnostic guidelines for Alzheimer's disease. Alzheimers Dement 2011;7:257-62.

[31] Sarazin M, de Souza LC, Lehéricy S, Dubois B. Clinical and research diagnostic criteria for Alzheimer's disease. Neuroimaging Clin N Am 2012;22:23-32. viii.

[32] Bertram L, Tanzi RE. The genetics of Alzheimer's disease. Prog Mol Biol Transl Sci 2012;107:79-100

[33] Bertram L, Lill CM, Tanzi RE. The genetics of Alzheimer disease: back to the future. Neuron 2010;68:270-81.

[34] Traynor BJ, Singleton AB. Nature versus nurture: death of a dogma, and the road ahead. Neuron 2010;68:196-200.

[35] Bertram L. Alzheimer's genetics in the GWAS era: a continuing story of 'replications and refutations'. Curr Neurol Neurosci Rep 2011;11: 246-53.

[36] Cruts M, Theuns J, Van Broeckhoven C. Locus-specific mutation databases for neurodegenerative brain diseases. Hum Mutat 2012;33:1340-4

[37] Steiner H, Fluhrer R, Haass C. Intramembrane proteolysis by gamma-secretase. J Biol Chem 2008;283:29627-31.

[38] Levy-Lahad E, Wasco W, Poorkaj P, Romano DM, Oshima J, Pettingell WH, et al. Candidate gene for the chromosome 1 familial Alzheimer's disease locus. Science 1995:269:973-7.

[39] Rogaev EI, Sherrington R, Rogaeva EA, Levesque G, Ikeda M, Liang Y, et al. Familial Alzheimer's disease in kindreds with missense mutations in a gene on chromosome 1 related to the Alzheimer's disease type 3 gene. Nature 1995;376:775-8

[40] Tanzi RE, Bertram L. Twenty years of the Alzheimer's disease amyloid hypothesis: a genetic perspective. Cell 2005;120:545-55.

[41] Pottier C, Hannequin D, Coutant S, Rovelet-Lecrux A, Wallon D, Rousseau S, et al. High frequency of potentially pathogenic SORL1 mutations in autosomal dominant early-onset Alzheimer disease. Mol Psychiatry 2012;17: 875-9.

[42] Strittmatter WJ, Saunders AM, Schmechel D, Pericak-Vance M, Enghild J, Salvesen GS, et al. Apolipoprotein E: high-avidity binding to beta-amyloid and increased frequency of type 4 allele in late-onset familial Alzheimer disease. Proc Natl Acad Sci USA 1993;90:1977-81.

[43] Corder EH, Saunders AM, Risch NJ, Strittmatter WJ, Schmechel DE, Gaskell Jr PC, et al. Protective effect of apolipoprotein E type 2 allele for late onset Alzheimer disease. Nat Genet 1994:7:180-4.

[44] Elshourbagy N, Liao W, Mahley R, Taylor J. Apolipoprotein E mRNA is abundant in the brain and adrenals, as well as in the liver, and is present in other peripheral tissues of rats and marmosets. Proc Natl Acad Sci USA $1985 ; 82: 203-7$

[45] Lin C, Xu Y, Wu J, Chan L. Immunoreactive apolipoprotein E is a widely distributed cellular protein. Immunohistochemical localization of apolipoprotein E in baboon tissues. J Clin Invest 1986;78:947-58. 
[46] Boyles J, Pitas R, Wilson E, Mahley R, Taylor J. Apolipoprotein E associated with astrocytic glia of the central nervous system and with nonmyelinating glia of the peripheral nervous system. J Clin Invest 1985;76:1501-13.

[47] Nakai M, Kawamata T, Taniguchi T, Maeda K, Tanaka C. Expression of apolipoprotein E mRNA in rat microglia. Neurosci Lett 1996;211:41-4.

[48] Youmans KL, Tai LM, Nwabuisi-Heath E, Jungbauer L, Kanekiyo T, Gan M, et al. APOE4-specific changes in $A \beta$ accumulation in a new transgenic mouse model of Alzheimer disease. J Biol Chem 2012;287:41774-86.

[49] Liu CC, Kanekiyo T, Xu H, Bu G, Apolipoprotein E. Alzheimer disease: risk, mechanisms and therapy. Nat Rev Neurol 2013;9:106-18.

[50] Thal DR, Papassotiropoulos A, Saido TC, Griffin WS, Mrak RE, Kölsch H, et al. Capillary cerebral amyloid angiopathy identifies a distinct APOE epsilon4associated subtype of sporadic Alzheimer's disease. Acta Neuropathol 2010;120:169-83.

[51] Bertram L, McQueen MB, Mullin K, Blacker D, Tanzi RE. Systematic metaanalyses of Alzheimer disease genetic association studies: the AlzGene database. Nat Genet 2007;39:17-23.

[52] Harold D, Abraham R, Hollingworth P, Sims R, Gerrish A, Hamshere ML, et al. Genome-wide association study identifies variants at CLU and PICALM associated with Alzheimer's disease. Nat Genet 2009;41:1088-93.

[53] Lambert JC, Heath S, Even G, Campion D, Sleegers K, Hiltunen M, et al. Genome-wide association study identifies variants at CLU and CR1 associated with Alzheimer's disease. Nat Genet 2009:41:1094-9.

[54] Seshadri S, Fitzpatrick AL, Ikram MA, DeStefano AL, Gudnason V, Boada M, et al. Genome-wide analysis of genetic loci associated with Alzheimer disease. J Am Med Assoc 2010;303:1832-40.

[55] Hollingworth P, Harold D, Sims R, Gerrish A, Lambert JC, Carrasquillo MM, et al. Common variants at ABCA7, MS4A6A/MS4A4E, EPHA1, CD33 and CD2AP are associated with Alzheimer's disease. Nat Genet 2011;43:429-35.

[56] Naj AC, Jun G, Beecham GW, Wang LS, Vardarajan BN, Buros J, et al. Common variants at MS4A4/MS4A6E, CD2AP, CD33 and EPHA1 are associated with late-onset Alzheimer's disease. Nat Genet 2011;43:436-41.

[57] Zetzsche T, Rujescu D, Hardy J, Hampel H. Advances and perspectives from genetic research: development of biological markers in Alzheimer's disease. Expert Rev Mol Diagn 2010;10:667-90.

[58] Jones L, Holmans PA, Hamshere ML, Harold D, Moskvina V, Ivanov D, et al. Genetic evidence implicates the immune system and cholesterol metabolism in the aetiology of Alzheimer's disease. PLoS ONE 2010;5:e13950.

[59] Jonsson T, Stefansson H, Steinberg S, Jonsdottir I, Jonsson PV, Snaedal J, et al. Variant of TREM2 associated with the risk of Alzheimer's disease. N Engl J Med 2013:368:107-16.

[60] Guerreiro R, Wojtas A, Bras J, Carrasquillo M, Rogaeva E, Majounie E, et al. TREM2 variants in Alzheimer's disease. N Engl J Med 2013;368:117-27.

[61] Neumann H, Daly MJ. Variant TREM2 as risk factor for Alzheimer's disease. N Engl J Med 2013;368:182-4.

[62] Zetterberg H, Tullhög K, Hansson O, Minthon L, Londos E, Blennow K. Low incidence of post-lumbar puncture headache in 1089 consecutive memory clinic patients. Eur Neurol 2010;63:326-30

[63] Andreasen N, Minthon L, Davidsson P, Vanmechelen E, Vanderstichele H, Winblad B, et al. Evaluation of CSF-tau and CSF-Abeta42 as diagnostic markers for Alzheimer disease in clinical practice. Arch Neurol 2001;58:373-9.

[64] Tabaraud F, Leman JP, Milor AM, Roussie JM, Barrière G, Tartary M, et al. Alzheimer CSF biomarkers in routine clinical setting. Acta Neurol Scand 2012;125:416-23.

[65] Dodel R, Rominger A, Bartenstein P, Barkhof F, Blennow K, Förster S, et al. Intravenous immunoglobulins for the treatment of mild to moderate Alzheimer's disease: a phase II, randomised, double-blind, placebo-controlled trial. Lancet Neurol 2013;12:233-43.

[66] Winblad B, Andreasen N, Minthon L, Floesser A, Imbert G, Dumortier T, et al. Safety, tolerability, and antibody response of active $A \beta$ immunotherapy with CAD106 in patients with Alzheimer's disease: randomised, double-blind, placebo-controlled, first-in-human study. Lancet Neurol 2012;11:597-604.

[67] Lannfelt L, Blennow K, Zetterberg H, Batsman S, Ames D, Harrison J, et al. Safety, efficacy, and biomarker findings of PBT2 in targeting A 3 as a modifying therapy for Alzheimer's disease: a phase IIa, double-blind, randomised, placebo-controlled trial. Lancet Neurol 2008;7:779-86.

[68] Vandermeeren M, Mercken M, Vanmechelen E, Six J, van de Voorde A, Martin JJ, et al. Detection of tau proteins in normal and Alzheimer's disease cerebrospinal fluid with a sensitive sandwich enzyme-linked immunosorbent assay. J Neurochem 1993;61:1828-34.

[69] Blennow K, Wallin A, Agren $H$, Spenger C, Siegfried J, Vanmechelen E. Tau protein in cerebrospinal fluid: a biochemical marker for axonal degeneration in Alzheimer disease. Mol Chem Neuropathol 1995;26:231-45.

[70] Motter R, Vigo-Pelfrey C, Kholodenko D, Barbour R, Johnson-Wood K, Galasko $\mathrm{D}$, et al. Reduction of beta-amyloid peptide42 in the cerebrospinal fluid of patients with Alzheimer's disease. Ann Neurol 1995;38:643-8.

[71] Blennow K. Cerebrospinal fluid protein biomarkers for Alzheimer's disease. NeuroRx 2004:1:213-25.

[72] Blennow K, Hampel H. Cerebrospinal fluid markers for incipient Alzheimer's disease. Lancet Neurol 2003;2:605-13.

[73] Hampel H, Teipel SJ, Fuchsberger T, Andreasen N, Wiltfang J, Otto M, et al. Value of CSF beta-amyloid1-42 and tau as predictors of Alzheimer's disease in patients with mild cognitive impairment. Mol Psychiatry 2004;9:705-10.

[74] Koopman K, Le Bastard N, Martin JJ, Nagels G, De Deyn PP, Engelborghs S Improved discrimination of autopsy-confirmed Alzheimer's disease (AD) from non-AD dementias using CSF P-tau(181P). Neurochem Int 2009:55:214-8.

[75] Shaw LM, Vanderstichele H, Knapik-Czajka M, Clark CM, Aisen PS, Petersen RC, et al. Cerebrospinal fluid biomarker signature in Alzheimer's disease neuroimaging initiative subjects. Ann Neurol 2009;65:403-13.

[76] Hansson O, Zetterberg H, Buchhave P, Londos E, Blennow K, Minthon L. Association between CSF biomarkers and incipient Alzheimer's disease in patients with mild cognitive impairment: a follow-up study. Lancet Neurol 2006:5:228-34.

[77] Snider BJ, Fagan AM, Roe C, Shah AR, Grant EA, Xiong C, et al. Cerebrospinal fluid biomarkers and rate of cognitive decline in very mild dementia of the Alzheimer type. Arch Neurol 2009;66:638-45.

[78] Visser PJ, Verhey F, Knol DL, Scheltens P, Wahlund LO, Freund-Levi Y, et al Prevalence and prognostic value of CSF markers of Alzheimer's disease pathology in patients with subjective cognitive impairment or mild cognitive impairment in the DESCRIPA study: a prospective cohort study. Lancet Neurol 2009;8:619-27.

[79] Mattsson N, Zetterberg H, Hansson O, Andreasen N, Parnetti L, Jonsson M et al. CSF biomarkers and incipient Alzheimer disease in patients with mild cognitive impairment. J Am Med Assoc 2009;302:385-93.

[80] Skoog I, Davidsson P, Aevarsson O, Vanderstichele H, Vanmechelen E, Blennow K. Cerebrospinal fluid beta-amyloid 42 is reduced before the onset of sporadic dementia: a population-based study in 85-year-olds. Dement Geriatr Cogn Disord 2003;15:169-76.

[81] Gustafson DR, Skoog I, Rosengren L, Zetterberg H, Blennow K. Cerebrospinal fluid beta-amyloid 1-42 concentration may predict cognitive decline in older women. J Neurol Neurosurg Psychiatry 2007;78:461-4.

[82] Stomrud E, Hansson O, Blennow K, Minthon L, Londos E. Cerebrospinal fluid biomarkers predict decline in subjective cognitive function over 3 years in healthy elderly. Dement Geriatr Cogn Disord 2007;24:118-24.

[83] Moonis M, Swearer JM, Dayaw MP, St George-Hyslop P, Rogaeva E, Kawarai T, et al. Familial Alzheimer disease: decreases in CSF Abeta42 levels precede cognitive decline. Neurology 2005;65:323-5.

[84] Ringman JM, Younkin SG, Pratico D, Seltzer W, Cole GM, Geschwind DH, et al. Biochemical markers in persons with preclinical familial Alzheimer disease. Neurology 2008;71:85-92.

[85] Bateman RJ, Xiong C, Benzinger TL, Fagan AM, Goate A, Fox NC, et al. Clinical and biomarker changes in dominantly inherited Alzheimer's disease. N Engl J Med 2012;367:795-804.

[86] Reiman EM, Quiroz YT, Fleisher AS, Chen K, Velez-Pardo C, Jimenez-Del-Rio $\mathrm{M}$, et al. Brain imaging and fluid biomarker analysis in young adults at genetic risk for autosomal dominant Alzheimer's disease in the presenilin 1 E280A kindred: a case-control study. Lancet Neurol 2012;11:1048-56.

[87] Tarawneh R, Holtzman DM. Biomarkers in translational research of Alzheimer's disease. Neuropharmacology 2010;59:310-22.

[88] Shoji M, Matsubara E, Kanai M, Watanabe M, Nakamura T, Tomidokoro Y, et al. Combination assay of CSF tau, A beta 1-40 and A beta 1-42(43) as a biochemical marker of Alzheimer's disease. J Neurol Sci 1998;158: 134-40.

[89] Welge V, Fiege O, Lewczuk P, Mollenhauer B, Esselmann H, Klafki HW, et al. Combined CSF tau, p-tau181 and amyloid-beta 38/40/42 for diagnosing Alzheimer's disease. J Neural Transm 2009;116:203-12.

[90] Fagan AM, Roe CM, Xiong C, Mintun MA, Morris JC, Holtzman DM. Cerebrospinal fluid tau/beta-amyloid(42) ratio as a prediction of cognitive decline in nondemented older adults. Arch Neurol 2007;64:343-9.

[91] Li G, Sokal I, Quinn JF, Leverenz JB, Brodey M, Schellenberg GD, et al. CSF tau/ Abeta42 ratio for increased risk of mild cognitive impairment: a follow-up study. Neurology 2007;69:631-9.

[92] Brys M, Pirraglia E, Rich K, Rolstad S, Mosconi L, Switalski R, et al. Prediction and longitudinal study of CSF biomarkers in mild cognitive impairment. Neurobiol Aging 2009;30:682-90.

[93] Riemenschneider M, Lautenschlager N, Wagenpfeil S, Diehl J, Drzezga A, Kurz A. Cerebrospinal fluid tau and beta-amyloid 42 proteins identify Alzheimer disease in subjects with mild cognitive impairment. Arch Neurol 2002:59:1729-34

[94] Blennow K, Zetterberg H, Fagan AM. Fluid biomarkers in Alzheimer disease. Cold Spring Harb Perspect Med 2012;2:a006221

[95] Maddalena A, Papassotiropoulos A, Muller-Tillmanns B, Jung HH, Hegi T, Nitsch RM, et al. Biochemical diagnosis of Alzheimer disease by measuring the cerebrospinal fluid ratio of phosphorylated tau protein to b-amyloid peptide 42. Arch Neurol 2003;60:1202-6.

[96] Zetterberg $\mathrm{H}$, Wahlund LO, Blennow K. Cerebrospinal fluid markers for prediction of Alzheimer's disease. Neurosci Lett 2003;352:67-9.

[97] Olsson A, Vanderstichele H, Andreasen N, de Meyer G, Wallin A, Holmberg B, et al. Simultaneous measurement of $\beta$-amyloid(1-42), tau and phosphorylated tau (Thr181) in cerebrospinal fluid by the xMAP technology. Clin Chem 2005;51:336-45

[98] Lewczuk P, Kornhuber J, Vanderstichele H, Vanmechelen E, Esselmann H, Bibl $\mathrm{M}$, et al. Multiplexed quantification of dementia biomarkers in the CSF of patients with early dementias and MCI: a multicenter study. Neurobiol Aging 2008;29:812-8.

[99] Jack Jr CR, Knopman DS, Jagust WJ, Shaw LM, Aisen PS, Weiner MW, et al Hypothetical model of dynamic biomarkers of the Alzheimer's pathological cascade. Lancet Neurol 2010;9:119-28.

[100] Buchhave P, Minthon L, Zetterberg H, Wallin AK, Blennow K, Hansson O. Cerebrospinal fluid levels of $\beta$-amyloid $1-42$, but not of tau, are fully changed 
already 5 to 10 years before the onset of Alzheimer dementia. Arch Gen Psychiatry 2012;69:98-106.

[101] van Rossum IA, Vos SJ, Burns L, Knol DL, Scheltens P, Soininen H, et al. Injury markers predict time to dementia in subjects with $\mathrm{MCI}$ and amyloid pathology. Neurology 2012;79:1809-16.

[102] Teunissen CE, Verwey NA, Kester MI, van Uffelen K, Blankenstein MA. Standardization of assay procedures for analysis of the CSF biomarkers amyloid $\beta((1-42))$ tau, and phosphorylated tau in Alzheimer's disease: report of an International Workshop. Int J Alzheimers Dis 2010. http:// dx.doi.org/10.4061/2010/635053. pii: 635053 .

[103] Vanderstichele H, Bibl M, Engelborghs S, Le Bastard N, Lewczuk P, Molinuevo JL, et al. Standardization of preanalytical aspects of cerebrospinal fluid biomarker testing for Alzheimer's disease diagnosis: a consensus paper from the Alzheimer's Biomarkers Standardization Initiative. Alzheimers Dement 2012;8:65-73

[104] Mattsson N, Zetterberg H. What is a certified reference material. Biomark Med 2012;6:369-70.

[105] Mattsson N, Zegers I, Andreasson U, Bjerke M, Blankenstein MA, Bowser R, et al. Reference measurement procedures for Alzheimer's disease cerebrospinal fluid biomarkers: definitions and approaches with focus on amyloid beta42. Biomark Med 2012;6:409-17.

[106] Mattsson N, Andreasson U, Carrillo MC, Persson S, Shaw LM, Zegers I, et al. Proficiency testing programs for Alzheimer's disease cerebrospinal fluid biomarkers. Biomark Med 2012;6:401-7.

[107] Mattsson N, Andreasson U, Persson S, Carrillo MC, Collins S, Chalbot S, et al. CSF biomarker variability in the Alzheimer's association quality contro program. Alzheimers Dement 2013;9:251-61.

[108] Fagan AM, Perrin RJ. Upcoming candidate cerebrospinal fluid biomarkers of Alzheimer's disease. Biomark Med 2012;6:455-76.

[109] Kaddurah-Daouk R, Kristal B, Weinshilboum R. Metabolomics: a globa biochemical approach to drug response and disease. Annu Rev Pharmacol Toxicol 2008;48:653-83

[110] Patel S, Shah RJ, Coleman P, Sabbagh M. Potential peripheral biomarkers for the diagnosis of Alzheimer's disease. Int J Alzheimers Dis 2011. http:// dx.doi.org/10.4061/2011/572495. pii: 572495.

[111] Kaiser E, Schoenknecht P, Kassner S, Hildebrandt W, Kinscherf R, Schroeder J. Cerebrospinal fluid concentrations of functionally important amino acids and metabolic compounds in patients with mild cognitive impairment and Alzheimer's disease. Neurodegener Dis 2010;7:251-9.

[112] Czech C, Berndt P, Busch K, Schmitz O, Wiemer J, Most V, et al. Metabolite profiling of Alzheimer's disease cerebrospinal fluid. PLoS ONE 2012; 7:e31501.

[113] van Oijen M, Hofman A, Soares HD, Koudstaal PJ, Breteler MM. Plasma Abeta(1-40) and Abeta(1-42) and the risk of dementia: a prospective case-cohort study. Lancet Neurol 2006;5:655-60.

[114] Mayeux R, Honig LS, Tang MX, Manly J, Stern Y, Schupf N, et al. Plasma A[beta]40 and A[beta]42 and Alzheimer's disease: relation to age, mortality, and risk. Neurology 2003;61:1185-90.

[115] Hansson O, Zetterberg H, Vanmechelen E, Vanderstichele H, Andreasson U, Londos E, et al. Evaluation of plasma Abeta(40) and Abeta(42) as predictors of conversion to Alzheimer's disease in patients with mild cognitive impairment. Neurobiol Aging 2010;31:357-67.

[116] Lopez OL, Kuller LH, Mehta PD, Becker JT, Gach HM, Sweet RA, et al. Plasma amyloid levels and the risk of $\mathrm{AD}$ in normal subjects in the cardiovascular health study. Neurology 2008;70:1664-71.

[117] Sundelof J, Giedraitis V, Irizarry MC, Sundstrom J, Ingelsson E, Ronnemaa E, et al. Plasma beta amyloid and the risk of Alzheimer disease and dementia in elderly men: a prospective, population-based cohort study. Arch Neurol 2008;65:256-63.

[118] Graff-Radford NR, Crook JE, Lucas J, Boeve BF, Knopman DS, Ivnik RJ, et al. Association of low plasma Abeta42/Abeta40 ratios with increased imminent risk for mild cognitive impairment and Alzheimer disease. Arch Neurol 2007;64:354-62.

[119] Yaffe K, Weston A, Graff-Radford NR, Satterfield S, Simonsick EM, Younkin SG, et al. Association of plasma beta-amyloid level and cognitive reserve with subsequent cognitive decline. J Am Med Assoc 2011;305:261-6.

[120] Mayeux R, Tang MX, Jacobs DM, Manly J, Bell K, Merchant C, et al. Plasma amyloid beta-peptide 1-42 and incipient Alzheimer's disease. Ann Neurol 1999;46:412-6

[121] Koyama A, Okereke OI, Yang T, Blacker D, Selkoe DJ, Grodstein F. Plasma amyloid-beta as a predictor of dementia and cognitive decline: a systematic review and meta-analysis. Arch Neurol 2012:69:824-31.

[122] Randall J, Mortberg E, Provuncher GK, Fournier DR, Duffy DC, Rubertsson S, et al. Tau proteins in serum predict neurological outcome after hypoxic brain injury from cardiac arrest: results of a pilot study. Resuscitation 2013;84:351-6.

[123] Hesse C, Rosengren L, Andreasen N, Davidsson P, Vanderstichele H, Vanmechelen E, et al. Transient increase in total tau but not phospho-tau in human cerebrospinal fluid after acute stroke. Neurosci Lett 2001;297:187-90.

[124] Rosén C, Hansson O, Blennow K, Zetterberg H. Fluid biomarkers in Alzheimer's disease - current concepts. Mol Neurodegener 2013;8:20.

[125] Zetterberg H, Wilson D, Andreasson U, Minthon L, Blennow K, Randall J, et al. Plasma tau levels in Alzheimer's disease. Alzheimers Res Ther 2013;5:9.

[126] Ray S, Reddy PJ, Jain R, Gollapalli K, Moiyadi A, Srivastava S. Proteomic technologies for the identification of disease biomarkers in serum: advances and challenges ahead. Proteomics 2011;11:2139-61.
[127] Lista S, Faltraco F, Prvulovic D, Hampel H. Blood and plasma-based proteomic biomarkerresearchin Alzheimer's disease Prog Neurobiol2013:101-102:1-17.

[128] Thambisetty M, Lovestone S. Blood-based biomarkers of Alzheimer's disease: challenging but feasible. Biomark Med 2010;4:65-79.

[129] Ray S, Britschgi M, Herbert C, Takeda-Uchimura Y, Boxer A, Blennow K, et al. Classification and prediction of clinical Alzheimer's diagnosis based on plasma signaling proteins. Nat Med 2007;13:1359-62.

[130] Hu WT, Holtzman DM, Fagan AM, Shaw LM, Perrin R, Arnold SE, et al. Plasma multianalyte profiling in mild cognitive impairment and Alzheimer disease. Neurology 2012;79:897-905.

[131] Wyss-Coray T. Inflammation in Alzheimer disease: driving force, bystander or beneficial response. Nat Med 2006;12:1005-15.

[132] Wyss-Coray T, Rogers J. Inflammation in Alzheimer disease-a brief review of the basic science and clinical literature. Cold Spring Harb Perspect Med 2012;2:a006346.

[133] Bjorkqvist M, Ohlsson M, Minthon L, Hansson O. Evaluation of a previously suggested plasma biomarker panel to identify Alzheimer's disease. PLoS ONE 2012; 7:e29868.

[134] Doecke JD, Laws SM, Faux NG, Wilson W, Burnham SC, Lam CP, et al. Bloodbased protein biomarkers for diagnosis of Alzheimer disease. Arch Neurol 2012;69:1318-25.

[135] O’Bryant SE, Xiao G, Barber R, Huebinger R, Wilhelmsen K, Edwards M, et al. A blood-based screening tool for Alzheimer's disease that spans serum and plasma: findings from TARC and ADNI. PLoS ONE 2011;6:e28092.

[136] Galasko D, Golde TE. Biomarkers for Alzheimer's disease in plasma, serum and blood - conceptual and practical problems. Alzheimers Res Ther 2013;5:10.

[137] Lista S, Faltraco F, Hampel H. Biological and methodical challenges of bloodbased proteomics in the field of neurological research. Prog Neurobiol 2013;101-102:18-34.

[138] Apweiler R, Aslanidis C, Deufel T, Gerstner A, Hansen J, Hochstrasser D, et al. Approaching clinical proteomics: current state and future fields of application in fluid proteomics. Clin Chem Lab Med 2009;47:724-44.

[139] Omenn GS, States DJ, Adamski M, Blackwell TW, Menon R, Hermjakob H, et al. Overview of the HUPO Plasma Proteome Project: results from the pilot phase with 35 collaborating laboratories and multiple analytical groups, generating a core dataset of 3020 proteins and a publicly-available database. Proteomics 2005;5:3226-45.

[140] Omenn GS. Data management and data integration in the HUPO plasma proteome project. Methods Mol Biol 2011;696:247-57.

[141] Clerx L, Visser PJ, Verhey F, Aalten P. New MRI markers for Alzheimer's disease: a meta-analysis of diffusion tensor imaging and a comparison with medial temporal lobe measurements. J Alzheimers Dis 2012;29:405-29.

[142] Fox NC, Black RS, Gilman S, Rossor MN, Griffith SG, Jenkins L, et al. Effects of Abeta immunization (AN1792) on MRI measures of cerebral volume in Alzheimer disease. Neurology 2005;64:1563-72.

[143] Jack Jr CR, Slomkowski M, Gracon S, Hoover TM, Felmlee JP, Stewart K, et al. MRI as a biomarker of disease progression in a therapeutic trial of milameline for AD. Neurology 2003;60:253-60.

[144] Wilkinson D, Fox NC, Barkhof F, Phul R, Lemming O, Scheltens P. Memantine and brain atrophy in Alzheimer's disease: a 1-year randomized controlled trial. J Alzheimers Dis 2012;29:459-69.

[145] Frisoni GB, Jack CR. Harmonization of magnetic resonance-based manual hippocampal segmentation: a mandatory step for wide clinical use. Alzheimers Dement 2011;7:171-4.

[146] Jack Jr CR, Barkhof F, Bernstein MA, Cantillon M, Cole PE, Decarli C. Steps to standardization and validation of hippocampal volumetry as a biomarker in clinical trials and diagnostic criterion for Alzheimer's disease. Alzheimers Dement 2011;7. 474-85.e4.

[147] De Souza LC, Chupin M, Bertoux M, Lehéricy S, Dubois B, Lamari F, et al. Is hippocampal volume a good marker to differentiate Alzheimer's disease from frontotemporal dementia. J Alzheimers Dis 2013;36:57-66.

[148] Laakso MP, Partanen K, Riekkinen P, Lehtovirta M, Helkala EL, Hallikainen M, et al. Hippocampal volumes in Alzheimer's disease, Parkinson's disease with and without dementia, and in vascular dementia: an MRI study. Neurology 1996;46:678-81.

[149] Hashimoto M, Kitagaki H, Imamura T, Hirono N, Shimomura T, Kazui H, et al. Medial temporal and whole-brain atrophy in dementia with Lewy bodies: a volumetric MRI study. Neurology 1998;51:357-62.

[150] Videbech P, Ravnkilde B. Hippocampal volume and depression: a metaanalysis of MRI studies. Am J Psychiatry 2004;161:1957-66.

[151] Fox NC, Crum WR, Scahill RI, Stevens JM, Janssen JC, Rossor MN. Imaging of onset and progression of Alzheimer's disease with voxel-compression mapping of serial magnetic resonance images. Lancet 2001;358:201-5.

[152] Schnack HG, van Haren NE, Hulshoff Pol HE, Picchioni M, Weisbrod M, Sauer $\mathrm{H}$, et al. Reliability of brain volumes from multicenter MRI acquisition: a calibration study. Hum Brain Map 2004;22:312-20.

[153] Smith AD, Smith SM, de Jager CA, Whitbread P, Johnston C, Agacinski G, et al. Homocysteine-lowering by B vitamins slows the rate of accelerated brain atrophy in mild cognitive impairment: a randomized controlled trial. PLoS ONE 2010;5:e12244.

[154] Ashburner J. A fast diffeomorphic image registration algorithm. Neuroimage 2007;38:95-113.

[155] Douaud G, Refsum H, de Jager CA, Jacoby R, Nichols TE, Smith SM, et al. Preventing Alzheimer's disease-related gray matter atrophy by B-vitamin treatment. Proc Natl Acad Sci USA 2013;110:9523-8. 
[156] Dyrba M, Ewers M, Wegrzyn M, Kilimann I, Plant C, Oswald A, et al. Robust automated detection of microstructural white matter degeneration in Alzheimer's disease using machine learning classification of multicenter DTI data. PLoS ONE 2013;8:e64925

[157] Plant C, Teipel SJ, Oswald A, Bohm C, Meindl T, Mourao-Miranda J, et al. Automated detection of brain atrophy patterns based on MRI for the prediction of Alzheimer's disease. Neuroimage 2010;50:162-74.

[158] Klöppel S, Stonnington CM, Chu C, Draganski B, Scahill RI, Rohrer JD, et al. Automatic classification of MR scans in Alzheimer's disease. Brain 2008;131:681-9.

[159] Zarow C, Vinters HV, Ellis WG, Weiner MW, Mungas D, White L, et al. Correlates of hippocampal neuron number in Alzheimer's disease and ischemic vascular dementia. Ann Neurol 2005;57:896-903.

[160] Bobinski M, de Leon MJ, Wegiel J, Desanti S, Convit A, Saint Louis LA, et al. The histological validation of post mortem magnetic resonance imaging-determined hippocampal volume in Alzheimer's disease. Neuroscience 2000;95:721-5.

[161] Freeman SH, Kandel R, Cruz L, Rozkalne A, Newell K, Frosch MP, et al. Preservation of neuronal number despite age-related cortical brain atrophy in elderly subjects without Alzheimer disease. J Neuropathol Exp Neurol 2008;67:1205-12.

[162] Grinberg LT, Amaro Jr E, da Silva AV, da Silva RE, Sato JR, dos Santos DD, et al. Improved detection of incipient vascular changes by a biotechnological platform combining post mortem MRI in situ with neuropathology. J Neurol Sci 2009;283:2-8.

[163] Murray ME, Bieniek KF, Banks Greenberg M, Dejesus-Hernandez M, Rutherford NJ, van Blitterswijk M, et al. Progressive amnestic dementia, hippocampal sclerosis, and mutation in C9ORF72. Acta Neuropathol 2013. http:// dx.doi.org/10.1007/s00401-013-1161-2.

[164] Villemagne VL, Burnham S, Bourgeat P, Brown B, Ellis KA, Salvado O, et al. Amyloid $\beta$ deposition, neurodegeneration, and cognitive decline in sporadic Alzheimer's disease: a prospective cohort study. Lancet Neurol 2013;12:357-67.

[165] Westerberg C, Mayes A, Florczak SM, Chen Y, Creery J, Parrish T, et al. Distinct medial temporal contributions to different forms of recognition in amnestic mild cognitive impairment and Alzheimer's disease. Neuropsychologia 2013. http://dx.doi.org/10.1016/j.neuropsychologia.2013.06.025.

[166] Morris JC, Roe CM, Grant EA, Head D, Storandt M, Goate AM, et al. Pittsburgh compound $\mathrm{B}$ imaging and prediction of progression from cognitive normality to symptomatic Alzheimer disease. Arch Neurol 2009;66:1469-75.

[167] Giannakopoulos P, Kovari E, Gold G, von Gunten A, Hof PR, Bouras C. Pathological substrates of cognitive decline in Alzheimer's disease. Front Neurol Neurosci 2009;24:20-9.

[168] Mueller SG, Weiner MW. Selective effect of age, Apo e4, and Alzheimer's disease on hippocampal subfields. Hippocampus 2009;19:558-64.

[169] Hanseeuw BJ, Van Leemput K, Kavec M, Grandin C, Seron X, Ivanoiu A. Mild cognitive impairment: differential atrophy in the hippocampal subfields. AJNR Am J Neuroradiol 2011;32:1658-61.

[170] Wisse LE, Gerritsen L, Zwanenburg JJ, Kuijf HJ, Luijten PR, Biessels GJ, et al. Subfields of the hippocampal formation at $7 \mathrm{~T}$ MRI: in vivo volumetric assessment. Neuroimage 2012:61:1043-9.

[171] Henry TR, Chupin M, Lehéricy S, Strupp JP, Sikora MA, Sha ZY, et al. Hippocampal sclerosis in temporal lobe epilepsy: findings at $7 \mathrm{~T}^{1}$. Radiology 2011;261:199-209.

[172] Kerchner GA, Deutsch GK, Zeineh M, Dougherty RF, Saranathan M, Rutt BK. Hippocampal CA1 apical neuropil atrophy and memory performance in Alzheimer's disease. Neuroimage 2012;63:194-202.

[173] Bartus RT, Dean RL, Pontecorvo MJ, Flicker C. The cholinergic hypothesis: a historical overview, current perspective, and future directions. Ann N Y Acad Sci 1985;444:332-58.

[174] Mesulam M. The cholinergic lesion of Alzheimer's disease: pivotal factor or side show. Learn Mem 2004;11:43-9.

[175] Teipel SJ, Flatz WH, Heinsen H, Bokde AL, Schoenberg SO, Stockel S, et al. Measurement of basal forebrain atrophy in Alzheimer's disease using MRI. Brain 2005;128:2626-44.

[176] Zaborszky L, Hoemke L, Mohlberg H, Schleicher A, Amunts K, Zilles K. Stereotaxic probabilistic maps of the magnocellular cell groups in human basal forebrain. Neuroimage 2008;42:1127-41.

[177] Teipel SJ, Buchert R, Thome J, Hampel H, Pahnke J. Development of Alzheimer-disease neuroimaging-biomarkers using mouse models with amyloidprecursor protein-transgene expression. Prog Neurobiol 2011;95:547-56.

[178] Teipel SJ, Kaza E, Hadlich S, Bauer A, Brüning T, Plath AS, et al. Automated detection of amyloid- $\beta$-related cortical and subcortical signal changes in a transgenic model of Alzheimer's disease using high-field MRI. J Alzheimers Dis 2011;23:221-37.

[179] Falangola MF, Dyakin VV, Lee SP, Bogart A, Babb JS, Duff K, et al. Quantitative MRI reveals aging-associated T2 changes in mouse models of Alzheimer's disease. NMR Biomed 2007;20:343-51.

[180] Lee SP, Falangola MF, Nixon RA, Duff K, Helpern JA. Visualization of betaamyloid plaques in a transgenic mouse model of Alzheimer's disease using MR microscopy without contrast reagents. Magn Reson Med 2004;52:53844.

[181] Bertrand A, Pasquier A, Petiet A, Wiggins C, Kraska A, Joseph-Mathurin N, et al. Micro-MRI study of cerebral aging: ex vivo detection of hippocampal subfield reorganization, microhemorrhages and amyloid plaques in mouse lemur primates. PLoS ONE 2013;8:e56593.
[182] Nakada T, Matsuzawa H, Igarashi H, Fujii Y, Kwee IL. In vivo visualization of senile-plaque-like pathology in Alzheimer's disease patients by MR microscopy on a 7 T system. J Neuroimaging 2008;18:125-9.

[183] Basser PJ, Jones DK. Diffusion-tensor MRI: theory, experimental design and data analysis - a technical review. NMR Biomed 2002;15:456-67.

[184] O’Dwyer L, Lamberton F, Bokde AL, Ewers M, Faluyi YO, Tanner C, et al Multiple indices of diffusion identifies white matter damage in mild cognitive impairment and Alzheimer's disease. PLoS ONE 2011;6:e21745.

[185] Hess CP. Update on diffusion tensor imaging in Alzheimer's disease. Magn Reson Imaging Clin N Am 2009;17:215-24.

[186] Chua TC, Wen W, Slavin MJ, Sachdev PS. Diffusion tensor imaging in mild cognitive impairment and Alzheimer's disease: a review. Curr Opin Neurol 2008;21:83-92.

[187] Bozzali M, Cherubini A. Diffusion tensor MRI to investigate dementias: a brief review. Magn Reson Imaging 2007;25:969-77.

[188] Pierpaoli C, Basser PJ. Toward a quantitative assessment of diffusion anisotropy. Magn Reson Med 1996;36:893-906.

[189] Jones DK. Studying connections in the living human brain with diffusion MRI. Cortex 2008;44:936-52.

[190] Takahashi M, Hackney DB, Zhang G, Wehrli SL, Wright AC, O’Brien WT, et al. Magnetic resonance microimaging of intraaxonal water diffusion in live excised lamprey spinal cord. Proc Natl Acad Sci USA 2002;99:16192-96.

[191] Liu Y, Spulber G, Lehtimäki KK, Könönen M, Hallikainen I, Gröhn H, et al. Diffusion tensor imaging and tract-based spatial statistics in Alzheimer's disease and mild cognitive impairment. Neurobiol Aging 2011;32:1558-71.

[192] Medina D, DeToledo-Morrell L, Urresta F, Gabrieli JD, Moseley M, Fleischman $D$, et al. White matter changes in mild cognitive impairment and AD: diffusion tensor imaging study. Neurobiol Aging 2006;27:663-72.

[193] Chua TC, Wen W, Chen X, Kochan N, Slavin MJ, Trollor JN, et al. Diffusion tensor imaging of the posterior cingulate is a useful biomarker of mild cognitive impairment. Am J Geriatr Psychiatry 2009;17:602-13.

[194] Huang J, Friedland RP, Auchus AP. Diffusion tensor imaging of normalappearing white matter in mild cognitive impairment and early Alzheimer disease: preliminary evidence of axonal degeneration in the temporal lobe. AJNR Am J Neuroradiol 2007;28:1943-8.

[195] Bozzali M, Falini A, Franceschi M, Cercignani M, Zuffi M, Scotti G, et al. White matter damage in Alzheimer's disease assessed in vivo using diffusion tensor magnetic resonance imaging. J Neurol Neurosurg Psychiatry 2002;72:742-6.

[196] Douaud G, Jbabdi S, Behrens TE, Menke RA, Gass A, Monsch AU, et al. DTI measures in crossing-fibre areas: increased diffusion anisotropy reveals early white matter alteration in $\mathrm{MCI}$ and mild Alzheimer's disease. Neuroimage 2011;55:880-90.

[197] Ennis DB, Kindlmann G. Orthogonal tensor invariants and the analysis of diffusion tensor magnetic resonance images. Magn Reson Med 2006;55:13646.

[198] Stricker NH, Schweinsburg BC, Delano-Wood L, Wierenga CE, Bangen KJ, Haaland KY, et al. Decreased white matter integrity in late-myelinating fiber pathways in Alzheimer's disease supports retrogenesis. Neuroimage 2009;45:10-6.

[199] Teipel SJ, Stahl R, Dietrich O, Schoenberg SO, Perneczky R, Bokde AL, et al. Multivariate network analysis of fiber tract integrity in Alzheimer's disease. Neuroimage 2007;34:985-95.

[200] Giannelli M, Belmonte G, Toschi N, Pesaresi I, Ghedin P, Traino AC, et al. Technical note: DTI measurements of fractional anisotropy and mean diffusivity at $1.5 \mathrm{~T}$ : comparison of two radiofrequency head coils with different functional designs and sensitivities. Med Phys 2011;38:3205-11.

[201] Sexton CE, Kalu UG, Filippini N, Mackay CE, Ebmeier KP. A meta-analysis of diffusion tensor imaging in mild cognitive impairment and Alzheimer's disease. Neurobiol Aging 2011;32(2322):e5-18.

[202] Teipel SJ, Wegrzyn M, Meindl T, Frisoni G, Bokde AL, Fellgiebel A, et al. Anatomical MRI and DTI in the diagnosis of Alzheimer's disease: a European Multicenter Study. J Alzheimers Dis 2012;31:S33-47.

[203] Alexander AL, Hasan KM, Lazar M, Tsuruda JS, Parker DL. Analysis of partial volume effects in diffusion-tensor MRI. Magn Reson Med 2001;45:770-80.

[204] Giannelli M, Toschi N, Passamonti L, Mascalchi M, Diciotti S, Tessa C. Diffusion kurtosis and diffusion-tensor MR imaging in Parkinson disease. Radiology 2012;265:645-6.

[205] Fieremans E, Jensen JH, Helpern JA. White matter characterization with diffusional kurtosis imaging. Neuroimage 2011;58:177-88.

[206] Jensen JH, Helpern JA. MRI quantification of non-Gaussian water diffusion by kurtosis analysis. NMR Biomed 2010;23:698-710.

[207] Hui ES, Cheung MM, Qi L, Wu EX. Advanced MR diffusion characterization of neural tissue using directional diffusion kurtosis analysis. Conf Proc IEEE Eng Med Biol Soc 2008;2008:3941-4. http://dx.doi.org/10.1109/ IEMBS.2008.4650072.

[208] Wedeen VJ, Wang RP, Schmahmann JD, Benner T, Tseng WY, Dai G, et al Diffusion spectrum magnetic resonance imaging (DSI) tractography of crossing fibers. Neuroimage 2008;41:1267-77.

[209] Alexander DC. Multiple-fiber reconstruction algorithms for diffusion MRI. Ann N Y Acad Sci 2005;1064:113-33.

[210] Assaf Y, Blumenfeld-Katzir T, Yovel Y, Basser PJ. AxCaliber: a method for measuring axon diameter distribution from diffusion MRI. Magn Reson Med 2008;59:1347-54.

[211] Assaf Y, Basser PJ. Composite hindered and restricted model of diffusion (CHARMED) MR imaging of the human brain. Neuroimage 2005;27: 48-58. 
[212] De Santis S, Gabrielli A, Bozzali M, Maraviglia B, Macaluso E, Capuani S Anisotropic anomalous diffusion assessed in the human brain by scalar invariant indices. Magn Reson Med 2011;65:1043-52.

[213] Alexander DC, Hubbard PL, Hall MG, Moore EA, Ptito M, Parker GJ, et al Orientationally invariant indices of axon diameter and density from diffusion MRI. Neuroimage 2010;52:1374-89.

[214] De Santis S, Gabrielli A, Palombo M, Maraviglia B, Capuani S. Non-Gaussian diffusion imaging: a brief practical review. Magn Reson Imaging 2011:29:1410-6.

[215] Iraji A, Davoodi-Bojd E, Soltanian-Zadeh H, Hossein-Zadeh GA, Jiang Q. Diffusion kurtosis imaging discriminates patients with white matter lesions from healthy subjects. Conf Proc IEEE Eng Med Biol Soc 2011;2011:2796-9. http://dx.doi.org/10.1109/IEMBS.2011.6090765.

[216] Falangola MF, Jensen JH, Babb JS, Hu C, Castellanos FX, Di Martino A, et al. Age-related non-Gaussian diffusion patterns in the prefrontal brain. J Magn Reson Imaging 2008;28:1345-50.

[217] Filippini N, MacIntosh BJ, Hough MG, Goodwin GM, Frisoni GB, Smith SM, et al. Distinct patterns of brain activity in young carriers of the APOE-epsilon4 allele. Proc Natl Acad Sci USA 2009;106:7209-14.

[218] Borghesani PR, Johnson LC, Shelton AL, Peskind ER, Aylward EH, Schellenberg GD, et al. Altered medial temporal lobe responses during visuospatial encoding in healthy APOE*4 carriers. Neurobiol Aging 2008;29:981-91.

[219] Bookheimer SY, Strojwas MH, Cohen MS, Saunders AM, Pericak-Vance MA, Mazziotta JC, et al. Patterns of brain activation in people at risk for Alzheimer's disease. N Engl J Med 2000;343:450-6.

[220] Sperling RA, Dickerson BC, Pihlajamaki M, Vannini P, LaViolette PS, Vitolo OV, et al. Functional alterations in memory networks in early Alzheimer's disease. Neuromol Med 2010;12:27-43.

[221] Sperling R. Functional MRI studies of associative encoding in normal aging, mild cognitive impairment, and Alzheimer's disease. Ann N Y Acad Sci 2007;1097:146-55.

[222] Hämäläinen A, Pihlajamäki M, Tanila H, Hänninen T, Niskanen E, Tervo S, et al. Increased fMRI responses during encoding in mild cognitive impairment. Neurobiol Aging 2007;28:1889-903.

[223] Golby A, Silverberg G, Race E, Gabrieli S, O’Shea J, Knierim K, et al. Memory encoding in Alzheimer's disease: an fMRI study of explicit and implicit memory. Brain 2005;128:773-87.

[224] Grön G, Bittner D, Schmitz B, Wunderlich AP, Tomczak R, Riepe MW. Hippocampal activations during repetitive learning and recall of geometric patterns. Learn Mem 2001;8:336-45.

[225] Petrella JR, Wang L, Krishnan S, Slavin MJ, Prince SE, Tran TT, et al. Cortical deactivation in mild cognitive impairment: high-field-strength functional MR imaging. Radiology 2007;245:224-35.

[226] Johnson SC, Schmitz TW, Moritz CH, Meyerand ME, Rowley HA, Alexander AL, et al. Activation of brain regions vulnerable to Alzheimer's disease: the effect of mild cognitive impairment. Neurobiol Aging 2006;27:1604-12.

[227] Celone KA, Calhoun VD, Dickerson BC, Atri A, Chua EF, Miller SL, et al. Alterations in memory networks in mild cognitive impairment and Alzheimer's disease: an independent component analysis. J Neurosci 2006;26:10222-31.

[228] Satterthwaite TD, Green L, Myerson J, Parker J, Ramaratnam M, Buckner RL Dissociable but inter-related systems of cognitive control and reward during decision making: evidence from pupillometry and event-related fMRI. Neuroimage 2007;37:1017-31.

[229] Vannini P, Hedden T, Becker JA, Sullivan C, Putcha D, Rentz D, et al. Age and amyloid-related alterations in default network habituation to stimulus repetition. Neurobiol Aging 2012;33:1237-52.

[230] Pihlajamäki M, O'Keefe K, O'Brien J, Blacker D, Sperling RA. Failure of repetition suppression and memory encoding in aging and Alzheimer's disease. Brain Imaging Behav 2011;5:36-44.

[231] Pihlajamäki M, O’Keefe K, Bertram L, Tanzi RE, Dickerson BC, Blacker D, et al. Evidence of altered posteromedial cortical FMRI activity in subjects at risk for Alzheimer disease. Alzheimer Dis Assoc Disord 2010;24: 28-36.

[232] Pihlajamäki M, DePeau KM, Blacker D, Sperling RA. Impaired medial tempora repetition suppression is related to failure of parietal deactivation in Alzheimer disease. Am J Geriatr Psychiatry 2008;16:283-92.

[233] Fleisher AS, Sherzai A, Taylor C, Langbaum JB, Chen K, Buxton RB. Resting-state BOLD networks versus task-associated functional MRI for distinguishing Alzheimer's disease risk groups. Neuroimage 2009;47: 1678-90.

[234] Greicius MD, Supekar K, Menon V, Dougherty RF. Resting-state functional connectivity reflects structural connectivity in the default mode network Cereb Cortex 2009;19:72-8.

[235] Wermke M, Sorg C, Wohlschläger AM, Drzezga A. A new integrative model of cerebral activation, deactivation and default mode function in Alzheimer's disease. Eur J Nucl Med Mol Imaging 2008;35:S12-24.

[236] Wang K, Liang M, Wang L, Tian L, Zhang X, Li K, et al. Altered functional connectivity in early Alzheimer's disease: a resting-state fMRI study. Hum Brain Map 2007;28:967-78

[237] Sorg C, Riedl V, Mühlau M, Calhoun VD, Eichele T, Läer L, et al. Selective changes of resting-state networks in individuals at risk for Alzheimer's disease. Proc Natl Acad Sci USA 2007;104:18760-65.

[238] Greicius MD, Srivastava G, Reiss AL, Menon V. Default-mode network activity distinguishes Alzheimer's disease from healthy aging: evidence from functional MRI. Proc Natl Acad Sci USA 2004;101:4637-42.
[239] Petrella JR, Sheldon FC, Prince SE, Calhoun VD, Doraiswamy PM. Default mode network connectivity in stable vs. progressive mild cognitive impairment. Neurology 2011;76:511-7.

[240] Kukolja J, Thiel CM, Fink GR. Cholinergic stimulation enhances neural activity associated with encoding but reduces neural activity associated with retrieval in humans. J Neurosci 2009;29:8119-28.

[241] Shanks MF, McGeown WJ, Forbes-McKay KE, Waiter GD, Ries M, Venneri A. Regional brain activity after prolonged cholinergic enhancement in early Alzheimer's disease. Magn Reson Imaging 2007;25:848-59.

[242] Goekoop R, Scheltens P, Barkhof F, Rombouts SA. Cholinergic challenge in Alzheimer patients and mild cognitive impairment differentially affects hippocampal activation-a pharmacological fMRI study. Brain 2006;129:141-57.

[243] Saykin AJ, Wishart HA, Rabin LA, Flashman LA, McHugh TL, Mamourian AC, et al. Cholinergic enhancement of frontal lobe activity in mild cognitive impairment. Brain 2004;127:1574-83.

[244] Rombouts SA, Barkhof F, Van Meel CS, Scheltens P. Alterations in brain activation during cholinergic enhancement with rivastigmine in Alzheimer's disease. J Neurol Neurosurg Psychiatry 2002;73:665-71.

[245] Sperling RA. The potential of functional MRI as a biomarker in early Alzheimer's disease. Neurobiol Aging 2011;32:S37-43.

[246] Yang X, Beason-Held L, Resnick SM, Landman BA. Biological parametric mapping with robust and non-parametric statistics. Neuroimage 2011;57:423-30.

[247] Casanova R, Srikanth R, Baer A, Laurienti PJ, Burdette JH, Hayasaka S, et al. Biological parametric mapping: a statistical toolbox for multimodality brain image analysis. Neuroimage 2007;34:137-43.

[248] Oakes TR, Fox AS, Johnstone T, Chung MK, Kalin N, Davidson RJ. Integrating VBM into the General Linear Model with voxelwise anatomical covariates. Neuroimage 2007:34:500-8.

[249] Orrù G, Pettersson-Yeo W, Marquand AF, Sartori G, Mechelli A. Using Support Machine to identify imaging biomarkers of neurological and psychiatric disease: a critical review. Neurosci Biobehav Rev 2012;36:1140-52.

[250] Zhang D, Shen D, Alzheimer's Disease Neuroimaging Initiative. Multi-modal multi-task learning for joint prediction of multiple regression and classification variables in Alzheimer's disease. Neuroimage 2012;59:895-907.

[251] Zhang D, Shen D, Alzheimer's Disease Neuroimaging Initiative. Predicting future clinical changes of MCI patients using longitudinal and multimodal biomarkers. PLoS ONE 2012;7:e33182.

[252] Magistretti PJ, Pellerin L. Cellular mechanisms of brain energy metabolism. Relevance to functional brain imaging and to neurodegenerative disorders. Ann N Y Acad Sci 1996;777:380-7.

[253] Zamrini E, De Santi S, Tolar M. Imaging is superior to cognitive testing for early diagnosis of Alzheimer's disease. Neurobiol Aging 2004;25: 685-91.

[254] Silverman DH, Small GW, Chang CY, Lu CS, Kung De Aburto MA, et al. Positron emission tomography in evaluation of dementia: regional brain metabolism and long-term outcome. J Am Med Assoc 2001;286:2120-7.

[255] Minoshima S, Foster NL, Sima AA, Frey KA, Albin RL, Kuhl DE. Alzheimer's disease versus dementia with Lewy bodies: cerebral metabolic distinction with autopsy confirmation. Ann Neurol 2001:50:358-65.

[256] Drzezga A, Grimmer T, Riemenschneider M, Lautenschlager N, Siebner H, Alexopoulus $\mathrm{P}$, et al. Prediction of individual clinical outcome in MCI by means of genetic assessment and (18)F-FDG PET. J Nucl Med 2005;46:162532.

[257] Mosconi L, Brys M, Glodzik-Sobanska L, De Santi S, Rusinek H, de Leon MJ. Early detection of Alzheimer's disease using neuroimaging. Exp Gerontol 2007:42:129-38.

[258] Scheef L, Spottke A, Daerr M, Joe A, Striepens N, Kölsch H, et al. Glucose metabolism, gray matter structure, and memory decline in subjective memory impairment. Neurology 2012;79:1332-9.

[259] Reiman EM, Caselli RJ, Yun LS, Chen K, Bandy D, Minoshima S, et al. Preclinical evidence of Alzheimer's disease in persons homozygous for the epsilon 4 allele for apolipoprotein E. N Engl J Med 1996;334:752-8.

[260] Hellwig S, Amtage F, Kreft A, Buchert R, Winz OH, Vach W, et al. [ ${ }^{18}$ F]FDG-PET is superior to $\left[{ }^{123} \mathrm{I}\right] \mathrm{IBZM}-\mathrm{SPECT}$ for the differential diagnosis of parkinsonism. Neurology 2012;79:1314-22.

[261] Lehmann M, Ghosh PM, Madison C, Laforce Jr R, Corbetta-Rastelli C, Weiner MW, et al. Diverging patterns of amyloid deposition and hypometabolism in clinical variants of probable Alzheimer's disease. Brain 2013;136:844-58.

[262] Teipel SJ, Willoch F, Ishii K, Bürger K, Drzezga A, Engel R, et al. Resting state glucose utilization and the CERAD cognitive battery in patients with Alzheimer's disease. Neurobiol Aging 2006;27:681-90.

[263] Reiman EM, Caselli RJ, Chen K, Alexander GE, Bandy D, Frost J. Declining brain activity in cognitively normal apolipoprotein E epsilon 4 heterozygotes: a foundation for using positron emission tomography to efficiently test treatments to prevent Alzheimer's disease. Proc Natl Acad Sci USA 2001;98: 3334-9.

[264] Perneczky R, Drzezga A, Diehl-Schmid J, Schmid G, Wohlschläger A, Kars S, et al. Schooling mediates brain reserve in Alzheimer's disease: findings of fluoro-deoxy-glucose-positron emission tomography. J Neurol Neurosurg Psychiatry 2006;77:1060-3.

[265] Förster S, Buschert VC, Buchholz HG, Teipel SJ, Friese U, Zach C, et al. Effects of a 6-month cognitive intervention program on brain metabolism in amnestic mild cognitive impairment and mild Alzheimer's disease. J Alzheimers Dis 2011;25:695-706. 
[266] Teipel SJ, Drzezga A, Bartenstein P, Möller HJ, Schwaiger M, Hampel H. Effects of donepezil on cortical metabolic response to activation during (18)FDG-PET in Alzheimer's disease: a double-blind cross-over trial. Psychopharmacology (Berlin) 2006;187:86-94.

[267] Klunk WE, Engler H, Nordberg A, Wang Y, Blomqvist G, Holt DP, et al. Imaging brain amyloid in Alzheimer's disease with Pittsburgh Compound-B. Ann Neurol 2004;55:306-19.

[268] Rowe CC, Villemagne VL. Brain amyloid imaging. J Nucl Med 2011;52:173340.

[269] Cselényi Z, Jönhagen ME, Forsberg A, Halldin C, Julin P, Schou M, et al. Clinical validation of ${ }^{18}$ F-AZD4694, an amyloid- $\beta$-specific PET radioligand. J Nucl Med 2012:53:415-24.

[270] Rowe CC.In: The Centiloid Scale: Standardization of Amyloid Imaging Measures. Abstract. Boston, MA, USA: Alzheimer Association International Conference; 2013.

[271] Ikonomovic MD, Klunk WE, Abrahamson EE, Mathis CA, Price JC, Tsopelas ND, et al. Post-mortem correlates of in vivo PiB-PET amyloid imaging in a typical case of Alzheimer's disease. Brain 2008;131:1630-45.

[272] Clark CM, Schneider JA, Bedell BJ, Beach TG, Bilker WB, Mintun MA, et al. Use of florbetapir-PET for imaging beta-amyloid pathology. J Am Med Assoc 2011;305:275-83.

[273] Thompson PW, Ye L, Morgenstern JL, Sue L, Beach TG, Judd DJ, et al. Interaction of the amyloid imaging tracer FDDNP with hallmark Alzheimer's disease pathologies. J Neurochem 2009;109:623-30.

[274] Foster ER, Campbell MC, Burack MA, Hartlein J, Flores HP, Cairns NJ, et al. Amyloid imaging of Lewy body-associated disorders. Mov Disord 2010;25:2516-23.

[275] Okello A, Koivunen J, Edison P, Archer HA, Turkheimer FE, Någren K, et al. Conversion of amyloid positive and negative MCI to AD over 3 years: an $11 \mathrm{C}$ PIB PET study. Neurology 2009;73:754-60.

[276] Nordberg A, Carter SF, Rinne J, Drzezga A, Brooks DJ, Vandenberghe R, et al. A European multicentre PET study of fibrillar amyloid in Alzheimer's disease. Eur J Nucl Med Mol Imaging 2013;40:104-14.

[277] Amariglio RE, Becker JA, Carmasin J, Wadsworth LP, Lorius N, Sullivan C, et al. Subjective cognitive complaints and amyloid burden in cognitively normal older individuals. Neuropsychologia 2012;50:2880-6.

[278] Reiman EM, Chen K, Liu X, Bandy D, Yu M, Lee W, et al. Fibrillar amyloid-beta burden in cognitively normal people at 3 levels of genetic risk for Alzheimer's disease. Proc Natl Acad Sci USA 2009;106:6820-5.

[279] Mintun MA, Larossa GN, Sheline YI, Dence CS, Lee SY, Mach RH, et al. [11 C]PIB in a nondemented population: potential antecedent marker of Alzheimer disease. Neurology 2006;67:446-52.

[280] Hedden T, Van Dijk KR, Becker JA, Mehta A, Sperling RA, Johnson KA, et al. Disruption of functional connectivity in clinically normal older adults harboring amyloid burden. J Neurosci 2009;29:12686-94.

[281] Selkoe DJ. Soluble oligomers of the amyloid beta-protein impair synaptic plasticity and behavior. Behav Brain Res 2008;192:106-13.

[282] Förster S, Grimmer T, Miederer I, Henriksen G, Yousefi BH, Graner P, et al. Regional expansion of hypometabolism in Alzheimer's disease follows amyloid deposition with temporal delay. Biol Psychiatry 2012;71:792-7.

[283] Rinne JO, Brooks DJ, Rossor MN, Fox NC, Bullock R, Klunk WE, et al. 11C-PiB PET assessment of change in fibrillar amyloid-beta load in patients with Alzheimer's disease treated with bapineuzumab: a phase 2, double-blind, placebo-controlled, ascending-dose study. Lancet Neurol 2010;9:363-72.

[284] Johnson KA, Minoshima S, Bohnen NI, Donohoe KJ, Foster NL, Herscovitch P, et al. Appropriate use criteria for amyloid PET: a report of the Amyloid Imaging Task Force, the Society of Nuclear Medicine and Molecular Imaging, and the Alzheimer's Association. J Nucl Med 2013:54:476-90.

[285] Jack Jr CR, Knopman DS, Jagust WJ, Petersen RC, Weiner MW, Aisen PS, et al. Tracking pathophysiological processes in Alzheimer's disease: an updated hypothetical model of dynamic biomarkers. Lancet Neurol 2013;12:207-16.

[286] Knopman DS, Jack Jr CR, Wiste HJ, Weigand SD, Vemuri P, Lowe VJ. Brain injury biomarkers are not dependent on $\beta$-amyloid in normal elderly. Ann Neurol 2012. http://dx.doi.org/10.1002/ana.23816.

[287] Sheline YI, Morris JC, Snyder AZ, Price JL, Yan Z, D’Angelo G, et al. APOE4 allele disrupts resting state fMRI connectivity in the absence of amyloid plaques or decreased CSF A 342 . J Neurosci 2010;30:17035-40.

[288] Okamura N, Furumoto S, Harada R, Tago T, Yoshikawa T, Fodero-Tavoletti M, et al. Novel ${ }^{18} \mathrm{~F}$-labeled arylquinoline derivatives for noninvasive imaging of tau pathology in Alzheimer disease. J Nucl Med 2013;54:1420-7.

[289] Micanovic C, Pal S. The diagnostic utility of EEG in early-onset dementia: a systematic review of the literature with narrative analysis. J Neural Transm 2013. http://dx.doi.org/10.1007/s00702-013-1070-5.

[290] Stam CJ. Use of magnetoencephalography (MEG) to study functional brain networks in neurodegenerative disorders. J Neurol Sci 2010;289:128-34.

[291] Babiloni C, Ferri R, Binetti G, Cassarino A, Dal Forno G, Ercolani M, et al. Fronto-parietal coupling of brain rhythms in mild cognitive impairment: a multicentric EEG study. Brain Res Bull 2006;69:63-73.

[292] Poil S, De Haan W, van der Flier WM, Mansvelder HD, Scheltens P, Linkenkaer-Hansen K. Integrative EEG biomarkers predict progression to Alzheimer's disease at the MCI stage. Front Aging Neurosci 2013;5:58.

[293] Luckhaus C, Grass-Kapanke B, Blaeser I, Ihl R, Supprian T, Winterer G, et al. Quantitative EEG in progressing vs. stable mild cognitive impairment $(\mathrm{MCI})$ : results of a 1-year follow-up study. Int J Geriatr Psychiatry 2008;23:1148-55.

[294] Grunwald M, Busse F, Hensel A, Kruggel F, Riedel-Heller S, Wolf H, et al. Correlation between cortical theta activity and hippocampal volumes in health, mild cognitive impairment, and mild dementia. J Clin Neurophysiol 2001; $18: 178-84$

[295] Jelic V, Johansson SE, Almkvist O, Shigeta M, Julin P, Nordberg A, et al Quantitative electroencephalography in mild cognitive impairment: longitudinal changes and possible prediction of Alzheimer's disease. Neurobiol Aging 2000;21:533-40.

[296] Dauwels J, Vialatte F, Musha T, Cichocki A. A comparative study of synchrony measures for the early diagnosis of Alzheimer's disease based on EEG. Neuroimage 2010:49:668-93.

[297] Stam CJ, Montez T, Jones BF, Rombouts SA, van der Made Y, Pijnenburg YA, et al. Disturbed fluctuations of resting state EEG synchronization in Alzheimer's disease. Clin Neurophysiol 2005;116:708-15.

[298] Drago V, Babiloni C, Bartrés-Faz D, Caroli A, Bosch B, Hensch T, et al. Disease tracking markers for Alzheimer's disease at the prodromal (MCI) stage. J Alzheimers Dis 2011;26:159-99.

[299] Jagust WJ, Landau SM, Shaw LM, Trojanowski JQ, Koeppe RA, Reiman EM, et al. Relationships between biomarkers in aging and dementia. Neurology 2009;73:1193-9.

[300] Rabinovici GD, Jagust WJ. Amyloid imaging in aging and dementia: testing the amyloid hypothesis in vivo. Behav Neurol 2009;21:117-28.

[301] Dubois B, Picard G, Sarazin M. Early detection of Alzheimer's disease: new diagnostic criteria. Dialogues Clin Neurosci 2009;11:135-9.

[302] Jackson CE, Snyder PJ. Electroencephalography and event-related potentials as biomarkers of mild cognitive impairment and mild Alzheimer's disease. Alzheimers Dement 2008;4:S137-43.

[303] Papaliagkas V, Kimiskidis V, Tsolaki M, Anogianakis G. Usefulness of eventrelated potentials in the assessment of mild cognitive impairment. BMC Neurosci 2008;9:107.

[304] Olichney JM, Taylor JR, Gatherwright J, Salmon DP, Bressler AJ, Kutas M, et al. Patients with $\mathrm{MCI}$ and N400 or P600 abnormalities are at very high risk for conversion to dementia. Neurology 2008;70:1763-70.

[305] Vincent A. Methods for improving the signal-to-noise ratio of endogenousevoked potentials. Integr Physiol Behav Sci 1992;27:54-65.

[306] Dickerson BC, Sperling RA. Large-scale functional brain network abnormalities in Alzheimer's disease: insights from functional neuroimaging. Behav Neurol 2009;21:63-75.

[307] Nisticò R, Pignatelli M, Piccinin S, Mercuri NB, Collingridge G. Targeting synaptic dysfunction in Alzheimer's disease therapy. Mol Neurobiol 2012;46:572-87.

[308] Kozauer N, Katz R. Regulatory innovation and drug development for earlystage Alzheimer's disease. N Engl J Med 2013;368:1169-71.

[309] Hampel H, Carrillo MC. Alzheimer's disease-modernizing concept, biological diagnosis and therapy. 1st ed. Advances in biological psychiatry, vol. 28, 1st ed. Basel: Karger; 2012.

[310] Noel-Storr AH, Flicker L, Ritchie CW, Nguyen GH, Gupta T, Wood P, et al. Systematic review of the body of evidence for the use of biomarkers in the diagnosis of dementia. Alzheimers Dement 2013;9:e96-105.

[311] Cortes-Blanco A, Prieto-Yerro C, Martinez-Lazaro R, Zamora J, Jiménez-Huete A, Haberkamp M. Florbetapir (18F) for brain amyloid imaging-abstract F304-01. Alzheimers Dement 2012;8:425-6.

[312] Sperling R, Johnson K. Biomarkers of Alzheimer disease: current and future applications to diagnostic criteria. Continuum (Minneapolis Minn) 2013;19:325-38.

[313] Richard E, Schmand BA, Eikelenboom P, Van Gool WA, Alzheimer's disease Neuroimaging Initiative. MRI and cerebrospinal fluid biomarkers for predicting progression to Alzheimer's disease in patients with mild cognitive impairment: a diagnostic accuracy study. Br Med J 2013. http://dx.doi.org/ 10.1136/bmjopen-2012-002541. pii: e002541.

[314] Prvulovic D, Hampel H. Ethical considerations of biomarker use in neurodegenerative diseases-a case study of Alzheimer's disease. Prog Neurobiol 2011:95:517-9.

[315] Hampel H, Wilcock G, Andrieu S, Aisen P, Blennow K, Broich K, et al. Biomarkers for Alzheimer's disease therapeutic trials. Prog Neurobiol 2011;95:579-93.

[316] Lu JX, Qiang W, Yau WM, Schwieters CD, Meredith SC, Tycko R. Molecular structure of $\beta$-amyloid fibrils in Alzheimer's disease brain tissue. Cell 2013;154:1257-68.

[317] Aguzzi A, Gitler AD. A template for new drugs against Alzheimer's disease. Cell 2013:154:1182-4.

[318] Zetterberg H, Smith DH, Blennow K. Biomarkers of mild traumatic brain injury in cerebrospinal fluid and blood. Nat Rev Neurol 2013;9 201-10.

[319] Andreasen N, Zetterberg H. Amyloid-related biomarkers for Alzheimer's disease. Curr Med Chem 2008;15:766-71

[320] Reiman EM, Langbaum JB, Fleisher AS, Caselli RJ, Chen K, Ayutyanont N, et al Alzheimer's Prevention Initiative: a plan to accelerate the evaluation of presymptomatic treatments. J Alzheimers Dis 2011;26:321-9.

[321] Sperling R, Donohue M, Aisen P. The A4 trial: anti-amyloid treatment of asymptomatic Alzheimer's disease. Abstract F3-04-01. Alzheimers Dement $2012 ; 8: 425-6$

[322] Rowe CC, Ellis KA, Rimajova M, Bourgeat P, Pike KE, Jones G, et al. Amyloid imaging results from the Australian Imaging, Biomarkers and Lifestyle (AIBL) study of aging. Neurobiol Aging 2010;31:1275-83.

[323] Solomon A, Kivipelto M, Soininen H. Prevention of Alzheimer's disease: moving backward through the lifespan. J Alzheimers Dis 2013;33: S465-9. 
[324] Vellas B, Aisen PS, Sampaio C, Carrillo M, Scheltens P, Scherrer B, et al. Prevention trials in Alzheimer's disease: an EU-US task force report. Prog Neurobiol 2011;95:594-600.

[325] Noorbakhsh F, Overall CM, Power C. Deciphering complex mechanisms in neurodegenerative diseases: the advent of systems biology. Trends Neurosci 2009;32:88-100.
[326] Miller JA, Oldham MC, Geschwind DH. A systems level analysis of transcriptional changes in Alzheimer's disease and normal aging. J Neurosci 2008;28:1410-20.

[327] Hallock P, Thomas MA. Integrating the Alzheimer's disease proteome and transcriptome: a comprehensive network model of a complex disease. OMICS 2012;16:37-49. 